\title{
Discrete DARBOUX TRANSFORMATIONS, THE DISCRETE-TIME TODA LATTICE, AND THE ASKEY-WILSON POLYNOMIALS
}

\author{
Vyacheslav Spiridonov and Alexei Zhedanov
}

\begin{abstract}
Consequences of the Darboux transformations for the finite-difference Schrödinger equations and three-term recurrence relations for orthogonal polynomials are considered. An equivalence of the chain of these transformations, or discrete dressing chain, to the discrete-time Toda lattice is established. A more fundamental discrete-time Volterra lattice consisting of one simple differencedifference nonlinear equation is found. Some simple similarity reductions of these lattices are described. A group-theoretical meaning of the Darboux transformations is illustrated on the example of Meixner polynomials. The general set of Askey-Wilson potentials is shown to define a class of solutions of the derived discrete-time equations. A subset of the latter potentials that can be obtained by dressing of the free discrete Schrödinger equation is characterized. A class of $q$-Racah polynomials for $q^{N}=1$ orthogonal with respect to a positive measure is obtained by undressing of the finite-dimensional Chebyshev polynomials.
\end{abstract}

\section{Introduction}

There are many common links between Darboux transformations $[2,10,16,18,20-24$, $27,33,34,44,48,50,53-55,62,63,81-83,85,86]$ and symmetry properties of the classical special functions $[25,75]$. Darboux transformations are very much related to the Bäcklund and dressing transformations of the theory of solitons [20,24,50,53, $54,76,81,85,86]$. Firstly, they form a core of the factorization method $[2,10,23$, $44,55,62,63,68]$; secondly, in the theory of orthogonal polynomials their analogs go back to Christoffel $[16,18,20,27,48,75,82]$; and thirdly, in numerical calculations of matrix eigenvalues, they appear in the procedure called the $L R$-algorithm $[27,83]$ (a modified form of which is known also as the $Q R$-algorithm). As in all these cases the underlying transformations share many common properties, we refer to them as Darboux transformations and add the adjective "discrete" in the context of finitedifference equations. Loosely speaking, the aim of the present paper is to describe the connections between these transformations and symmetries of special functions in the example of a discrete Schrödinger equation and three-term recurrence relations for orthogonal polynomials. Since the subject lies at the cross point of various fields, we provide review material directed to non-experts. In particular, because the Darboux technique is developed best for the ordinary differential Schrödinger equation (1.1), we start from a brief summary of the corresponding results.

Let us pick out the particular solution $\phi(x)$ of the equation

$$
H \psi(x) \equiv-\psi^{\prime \prime}(x)+u(x) \psi(x)=\lambda \psi(x),
$$

Received June 20, 1994, revised January 20, 1995.

1991 Mathematics Subject Classification. 33D45, 35Q51, 58F07.

Key words and phrases: Darboux transformations, orthogonal polynomials, solitons, dressing, factorization method, roots of unity. 
where $u(x)$ is some smooth real potential, corresponding to a fixed eigenvalue of the spectral parameter, $\lambda=\mu$, i.e., $H \phi(x)=\mu \phi(x)$. The Darboux transformation is defined for the entire class of solutions of (1.1) by

$$
\tilde{\psi}(x)=R \psi(x)
$$

where $R$ is the first-order differential operator

$$
R=\frac{d}{d x}-\frac{\phi^{\prime}(x)}{\phi(x)}
$$

The result of the transformation is not equal to zero whenever $\psi(x) \neq 0, \phi(x)$. The relation (1.2) defines a set of wave functions $\tilde{\psi}(x)$ satisfying the new Schrödinger equation

$$
\tilde{H} \tilde{\psi}(x)=-\tilde{\psi}^{\prime \prime}(x)+\tilde{u}(x) \tilde{\psi}(x)=\lambda \tilde{\psi}(x), \quad \tilde{u}=u-2 \frac{d^{2}}{d x^{2}} \ln \phi(x),
$$

and the eigenvalue of $\tilde{\psi}(x)$ coincides with $\lambda$. Suppose that (1.1) is exactly solvable, i.e., the solutions for it are expressed in terms of known special functions. Then (1.4) is exactly solvable as well. This property of the Darboux transformation is a crucial one, since it allows us to construct more and more complicated potentials step by step in an iterative way.

The relationship between Darboux transformations and the factorization method $[44,62,63]$ is given as follows. If one considers the operator which is formally conjugate to $R$,

$$
L=-\frac{d}{d x}-\frac{\phi^{\prime}(x)}{\phi(x)},
$$

which maps eigenfunctions of $\tilde{H}$ onto those of $H$, then

$$
H=L R+\mu, \quad \tilde{H}=R L+\mu,
$$

which constitutes a representation (factorization) of the second-order differential operators as products of the first-order ones. At the operator level, the Darboux transformation is equivalent to the intertwining relations

$$
\tilde{H} R=R H, \quad L \tilde{H}=H L
$$

where $H$ and $\tilde{H}$ are the old and new Hamiltonians, respectively. It is easy to see from (1.7) that the spectral properties of operators $\tilde{H}$ and $H$ are very close to each other.

Let $u(x)$ be a bounded function for finite values of $x$. Then, the singularities of a new potential $\tilde{u}(x)$ are determined by zeros and singularities of the function $\phi(x)$. Since $u(x)$ is regular, the wave functions may be singular only at infinity. Let $H$ have only a discrete spectrum. Whenever $\mu>\lambda_{\min }$ where $\lambda_{\min }$ is the lowest discrete eigenvalue of $H$, the function $\phi(x)$ has a zero, and thus, $\tilde{u}(x)$ has a $1 / x^{2}$ singularity. This may lead to a strong difference in the spectral properties of $H$ and $\tilde{H}$, due to the different domains of definition of these operators and $R$.

If $\mu=\lambda_{\min }$ and $\phi(x)$ is a ground state wave function, then the spectrum of $\tilde{H}$ coincides with that of $H$ with the first level missing, i.e., by this procedure one removes the lowest level. If $\mu<\lambda_{\min }$, then it is possible to choose nodeless $\phi(x)$ such that $1 / \phi(x)$ will be normalizable, and the spectrum of $\tilde{H}$ then contains one level more than that of $H$, with an additional eigenvalue equal to $\mu$. In the latter two cases, one "undresses" and "dresses" respectively, the spectrum of a given potential. The undressing is defined uniquely, whereas the dressing introduces at each step an additional parameter entering the general solution of (1.1) for $\lambda=\mu$. Therefore, the result of removal and 
restoration of the lowest level gives, in general, a potential different from the original one. If $\mu<\lambda_{\min }$ and $\phi(x)$ does not have zeros, but $1 / \phi$ is not normalizable, then the spectra of $H$ and $\tilde{H}$ coincide exactly, i.e., one gets completely isospectral operators.

The intertwining relations (1.7) have a universal character. They were applied to the operators of a very general form by Zakharov and Shabat $[85,86]$, who introduced the term "dressing". In this paper, we consider the dressing technique for the Hamiltonian operators $H$ which are tridiagonal in some basis $|n\rangle$ of a given linear space

$$
H|n\rangle=|n+1\rangle+u_{n}|n-1\rangle+b_{n}|n\rangle
$$

where the discrete index $n$ labels the basis and real discrete "potentials" $u_{n}$ and $b_{n}$. For the moment, we do not specify either the range of $n$ or the nature of the states $|n\rangle$. One can imagine the $|n\rangle$ 's as columns with constant elements on the $n$-th site and zeros in other places. In this case, $H$ is simply a Jacobian matrix of an unspecified size.

Discrete Darboux transformations (DDT) were considered in detail by Geronimus $[33,34]$ without using that name, and more recently by Matveev and Salle $[53,54]$ in the context of the ordinary Toda equations [76] and their hierarchic generalizations. In $[53,54]$, DDT were used in the construction of new solutions of the Toda lattice from a set of given ones, but the question of the possibility of generating all exactly solvable potentials with the help of the dressing procedure was not analyzed. In this respect, the Wronskian approach of $[53,54]$ is not appropriate if one is interested in the system of functions appearing after the infinite number of DDT applied to some simple initial objects. A set of exact solutions of the Toda lattice described in [51, 87] shows the complexity of the problem of classification of integrable potentials. However, the dressing techniques suggest that one should try to create all such systems from the free motion ones.

The power of Darboux transformations was demonstrated by recent developments in the theory of a one-dimensional Schrödinger equation (1.1). Namely, a large class of potentials arising from an infinite number of application of the transformations (1.2) to the zero potential was described in [36, 59,64,69]. The use of Darboux transformations in the form of a dressing chain, which was analyzed to some extent already in [44], allows us to define families of Schrödinger operators and special functions obeying certain fixed symmetry properties. In particular, the set of self-similar potentials of $[64,69]$ is related to the $q$-deformed Lie algebraic structures and $q$-Painlevé equations [69]. An analogous class of the lattice Schrödinger operators, or tridiagonal operators (1.8) obeying the ordinary and $q$-deformed ladder relations, has been described in [70]. In this work, we present further results in this direction.

It should be noted that although a very close relationship between scattering theory and orthogonal polynomials has been exhibited in [12,30,31], no complete unification scheme has yet been developed. Moreover, the standard scattering theory is applicable only to a very restricted class of potentials. The dressing technique provides an adequate formalism for going beyond this restricted class, and this work may be considered as a step towards the unified picture of special functions of mathematical physics and the theory of solitons. It is worth mentioning that many consequences of DDT were already used in the theory of orthogonal polynomials. In this respect, the closest approach to the general discrete dressing technique has been reached in the works of Geronimus [33,34] and in the later papers by Chihara and Uvarov [16-18, 82] (see also $[14,15,29,39,47]$ ).

The paper is organized as follows. In Section 2, we derive Darboux transformations for the second-order finite-difference operator (1.8). In Section 3, we analyze results of 
the application of single or special double DDT to the simplest (free) system defined by the choice $u_{n}=1, b_{n}=0$. In Section 4 , we analyze two successive Darboux transformations with one and the same eigenvalue. A discrete-time Toda lattice (DTTL) and its relationship to the discrete dressing chain are discussed in Section 5. A parallel discussion of the Volterra lattice is given. The special similarity solutions of the DTTL are considered in Section 6, and in Section 7, the dynamics of DDT for the Meixner set of orthogonal polynomials of a discrete variable is analyzed. The general set of Askey-Wilson polynomials is considered in Section 8, where we show that it provides a class of solutions of the DTTL. The main new results of the paper are contained in Section 5 (equivalence of the discrete dressing chain to DTTL and new simple form of the discrete-time Volterra lattice), Section 6 (description of the similarity ansatz for the discrete-time Volterra lattice giving the Askey-Wilson polynomial recurrence coefficients), and Section 8 (description of the new $q$-Racah polynomials at $q^{N}=1$ orthogonal with respect to a positive measure).

\section{Darboux transformations for the discrete Schrödinger equation}

Throughout this paper, we use the language of non-relativistic quantum mechanics, because of its universality. Consider a Hilbert space $l^{2}(\Gamma)$ with orthonormal basis vectors $|n\rangle, n \in \Gamma$, which we call the basis states, $\langle n \mid m\rangle=\delta_{n m}$. These states are assumed to be eigenvectors of some self-adjoint operator. When $\hat{x}|n\rangle=n|n\rangle$ where $\hat{x}$ is an abstract coordinate operator on a lattice $\Gamma$, the coefficients $\psi_{n}$ in the expansion of an arbitrary state from $l^{2}(\Gamma)$ over $|n\rangle,|\Phi\rangle=\sum_{n} \psi_{n}|n\rangle$, play the role of a discrete analog of the Schrödinger wave function in coordinate representation. In this case, the operator $H$ (1.8) represents a discretized version of some Sturm-Liouville operator. However, one can equally think of the set $|n\rangle$ as formed by the discrete spectrum states of a usual differential operator. For example, the relations (1.8) may be enforced by some (nonlinear) symmetry algebra formed by $H$ together with the operator which determines the states $|n\rangle$. For our purposes, the origin of basic tridiagonality (1.8) does not play any particular role. The finite-difference operator (1.8) may be considered as well over the Hilbert space $L^{2}(\mathbf{R}), n \in \mathbf{R}$, but we do not consider this case below.

The eigenvalue problem for the operator (1.8), $H|\Phi\rangle=\lambda|\Phi\rangle$, is reduced to

$$
u_{n+1} \psi_{n+1}(\lambda)+\psi_{n-1}(\lambda)+b_{n} \psi_{n}(\lambda)=\lambda \psi_{n}(\lambda)
$$

which we call the discrete Schrödinger equation (DSE). There is a large class of boundary conditions which could be imposed upon (2.1) and lead to non-trivial spectral problems. For example, orthogonal polynomials of $\lambda$ of unlimited degree are defined by (2.1) for some potentials $u_{n}, b_{n}$, provided that

$$
\psi_{-1}=0, \quad \psi_{0}=1 \quad \text { or } \quad \psi_{0}=1, \quad \psi_{1} \propto \lambda .
$$

Then the integer index $n=0,1, \ldots$ enumerates the degree of the polynomials. When $u_{n}>0$, by the renormalization of the basis $|n\rangle$, one can always transform the Hamiltonian $H$ to a formal Hermitian form (in the usual matrix sense) and obtain

$$
\sqrt{u_{n+1}} \chi_{n+1}+\sqrt{u_{n}} \chi_{n-1}+b_{n} \chi_{n}=\lambda \chi_{n}
$$

however, it is more convenient to work with (1.8) and (2.1).

We are seeking a discrete Darboux transformation (DDT) in the form

$$
R|n\rangle=A_{n}|n-1\rangle+B_{n}|n\rangle
$$

where $A_{n}$ and $B_{n}$ are unknown coefficients to be found from the basic relation (1.7). Substituting (2.4) and (1.8) into (1.7) and equating coefficients in front of the linearly 
independent states, we obtain the relations

$$
\begin{gathered}
B_{n+1}=B_{n}, \\
A_{n} \tilde{u}_{n-1}=A_{n-1} u_{n}, \\
A_{n}+B_{n} \tilde{b}_{n}=A_{n+1}+B_{n} b_{n}, \\
A_{n} \tilde{b}_{n-1}+B_{n} \tilde{u}_{n}=A_{n} b_{n}+B_{n-1} u_{n},
\end{gathered}
$$

where $\tilde{u}_{n}$ and $\tilde{b}_{n}$ are new potentials of the Hamiltonian $\tilde{H}$.

From (2.5), we see that $B_{n}=$ constant. Without loss of generality, we can set $B_{n}=1$. Indeed, the operator $R$ can be multiplied by an arbitrary constant without destroying the basic relation (1.7). Then one can express $\tilde{u}_{n}$ and $\tilde{b}_{n}$ in terms of old potentials from (2.7) and (2.8) [53, 54], namely

$$
\begin{gathered}
\tilde{u}_{n}=u_{n+1} A_{n} / A_{n+1}, \\
\tilde{b}_{n}=b_{n}+A_{n+1}-A_{n} .
\end{gathered}
$$

Substituting (2.9) and (2.10) into (2.8), we obtain

$$
A_{n}-A_{n-1}+b_{n-1}-b_{n}+\frac{u_{n+1}}{A_{n+1}}-\frac{u_{n}}{A_{n}}=0
$$

which has the first integral

$$
\frac{u_{n+1}}{A_{n+1}}+A_{n}-b_{n}=-\mu
$$

where $\mu$ is some constant. This equation (2.12) is a discrete analog of the Riccati equation; hence, it can be linearized. Indeed, the substitution

$$
A_{n}=-\phi_{n-1} / \phi_{n}
$$

transforms (2.12) to the form

$$
u_{n+1} \phi_{n+1}+\phi_{n-1}+b_{n} \phi_{n}=\mu \phi_{n} .
$$

The constant $\mu$ is seen to play the role of a spectral parameter of the auxiliary DSE (2.14), whereas $\phi_{n}$ is some solution of this equation.

Using (2.13), we can rewrite (2.9) and (2.10) in a form analogous to (1.4)

$$
\begin{gathered}
\tilde{u}_{n}=u_{n+1} \frac{\phi_{n-1} \phi_{n+1}}{\phi_{n}^{2}}, \\
\tilde{b}_{n}=b_{n}-\frac{\phi_{n}}{\phi_{n+1}}+\frac{\phi_{n-1}}{\phi_{n}} .
\end{gathered}
$$

Consider also a transformation of the wave functions: $|\tilde{\Phi}\rangle=\sum_{n} \tilde{\psi}_{n}|n\rangle=R|\Phi\rangle$. Using (2.4) and (2.13) one finds

$$
\tilde{\psi}_{n}=\psi_{n}-\frac{\phi_{n}}{\phi_{n+1}} \psi_{n+1}
$$

The formulae (2.14)-(2.17) give a complete description of DDT for DSE. Whenever $|\Phi\rangle$ is an eigenstate of $H$ with the eigenvalue $\lambda$, the function $|\tilde{\Phi}\rangle=R|\Phi\rangle$ is an eigenstate of the Hamiltonian $\tilde{H}$ with the same eigenvalue, $\tilde{H} R|\Phi\rangle=\lambda R|\Phi\rangle$, which follows from the intertwining relation $\tilde{H} R=R H$. This means that the $\tilde{\psi}_{n}$ satisfy the equation

$$
\tilde{u}_{n+1} \tilde{\psi}_{n+1}+\tilde{\psi}_{n-1}+\tilde{b}_{n} \tilde{\psi}_{n}=\lambda \tilde{\psi}_{n} \text {. }
$$


We conclude that from a given solvable DSE (2.1) and a particular solution $\phi_{n}$ corresponding to some fixed value of the spectral parameter $\lambda=\mu$, one can construct another solvable DSE.

It is easy to verify the reciprocal intertwining relation $L \tilde{H}=H L$ where

$$
L|n\rangle=C_{n}|n\rangle+|n+1\rangle, \quad C_{n}=u_{n+1} / A_{n+1} .
$$

The operator $L$ obviously provides the reciprocal Darboux transformation from the eigenfunctions of $\tilde{H}$ to those of $H$ and leads to the identities (1.6), which now constitute a factorization of the second-order finite-difference operators as products of the firstorder ones.

Consider two succeeding Darboux transformations,

$$
R_{2} R_{1} H=R_{2} \tilde{H} R_{1}=\tilde{\tilde{H}} R_{2} R_{1}
$$

where $R_{1}$ and $R_{2}$ correspond to DDT with $\left(\mu, \phi_{n}\right)$ and with $\left(\tilde{\mu}, \tilde{\phi}_{n}\right)$, respectively. Then $\tilde{\phi}_{n}$ satisfies the DSE

$$
\tilde{u}_{n+1} \tilde{\phi}_{n+1}+\tilde{\phi}_{n-1}+\tilde{b}_{n} \tilde{\phi}_{n}=\tilde{\mu} \tilde{\phi}_{n}
$$

whose general solution for $\tilde{\mu} \neq \mu$ is

$$
\tilde{\phi}_{n}=W_{n+1} / \phi_{n+1}
$$

where

$$
W_{n} \equiv \phi_{n} \zeta_{n-1}-\phi_{n-1} \zeta_{n}
$$

is a discrete analog of the Wronskian and $\zeta_{n}$ is a general solution of the initial DSE (2.1) with eigenvalue $\lambda=\tilde{\mu}$. For the potentials $\tilde{\tilde{u}}_{n}$ and $\tilde{\tilde{b}}_{n}$, we then have

$$
\begin{gathered}
\tilde{\tilde{u}}_{n}=\tilde{u}_{n+1} \frac{\tilde{\phi}_{n-1} \tilde{\phi}_{n+1}}{\tilde{\phi}_{n}^{2}}=u_{n+2} \frac{W_{n} W_{n+2}}{W_{n+1}^{2}} \\
\tilde{\tilde{b}}_{n}=b_{n}+\frac{\phi_{n-1}}{\phi_{n}}-\frac{\phi_{n}}{\phi_{n+1}}+\frac{\tilde{\phi}_{n-1}}{\tilde{\phi}_{n}}-\frac{\tilde{\phi}_{n}}{\tilde{\phi}_{n+1}} .
\end{gathered}
$$

There are cases when separate transformations $R_{1,2}$ map some normalizable eigenstates of the Hamiltonian $H$ to the unnormalizable ones, but the product $R_{2} R_{1}$ is a well-defined operator.

For the particular case when $\mu=\tilde{\mu}$, if we assume that $\tilde{\phi}_{n}$ is given by $(2.21)$, where $\zeta_{n}$ is a solution of (2.14) linearly independent from $\phi_{n}$, then $W_{n+1} u_{n+1}=W_{n}$; thus

$$
\tilde{\tilde{u}}_{n}=u_{n+1}, \quad \tilde{\tilde{b}}_{n}=b_{n+1}, \quad \tilde{\tilde{\psi}}_{n}=(\lambda-\mu) \psi_{n+1} .
$$

This gives an inversion of the formula (2.17)

$$
\psi_{n}=\frac{1}{\lambda-\mu}\left(\tilde{\psi}_{n-1}-\frac{\tilde{\phi}_{n-1}}{\tilde{\phi}_{n}} \tilde{\psi}_{n}\right) .
$$

When $\tilde{\psi}_{n}(\lambda)$ are orthogonal polynomials and $\tilde{\phi}_{n}(\mu)=\tilde{\psi}_{n}(\mu)$, the corresponding $\psi_{n}(\lambda ; \mu)$ are called the kernel polynomials [17], which are nothing other than the reciprocal Darboux transforms of a given set of the polynomials (in our notations $n$ is not a power of polynomials). A richer situation emerges when one takes the general solution of the equation (2.20) with $\tilde{\mu}=\mu$ as an auxiliary wave function for the second DDT. This possibility is considered in Section 4. 
Another simplified picture arises for $b_{n}=0, \tilde{\mu}=-\mu$. In this case, one can choose the solution $\zeta_{n}=(-1)^{n} \phi_{n}$, so that $\tilde{\phi}_{n}=2(-1)^{n} \phi_{n}$, and

$$
\tilde{\tilde{u}}_{n}=u_{n+2} \frac{\phi_{n-1} \phi_{n+2}}{\phi_{n} \phi_{n+1}}, \quad \tilde{\tilde{b}}_{n}=0 .
$$

As a result, one has

$$
R_{2} R_{1}|n\rangle=|n\rangle-\frac{\phi_{n-2}}{\phi_{n}}|n-2\rangle,
$$

which corresponds to the following transformation law for the wave functions

$$
\tilde{\tilde{\psi}}_{n}=\psi_{n}-\phi_{n} \psi_{n+2} / \phi_{n+2} \text {. }
$$

Note that in this case, the double DDT, $R_{2} R_{1}$, does not add the diagonal term to $H$, i.e., $\tilde{\tilde{b}}_{n} \equiv 0$. It is well known that the spectral problems $(2.3)$ with $b_{n} \neq 0$ and $b_{n}=0$ are associated with the Toda and Volterra lattices, respectively, whose continuous-time dynamics describe isospectral deformations of the Hamiltonian $H$ [76].

\section{One-soliton potentials and the related orthogonal polynomials}

This section contains an elementary exposition of the relations between the soliton potentials and orthogonal polynomials. Consider the simplest possible potentials, $u_{n} \equiv 1, b_{n} \equiv 0$, which correspond to the free discrete Schrödinger equation

$$
\phi_{n+1}+\phi_{n-1}=\mu \phi_{n}
$$

with the following general solution:

(a) for $2<\mu<\infty$, we set $\mu=2 \cosh \omega, 0<\omega<\infty$, and write

$$
\phi_{n}=\alpha \cosh (\omega n+\beta) ;
$$

(b) for $-\infty<\mu<-2$, we set $\mu=-2 \cosh \omega$, and write

$$
\phi_{n}=\alpha(-1)^{n} \cosh (\omega n+\beta) ;
$$

(c) for $-2<\mu<2$, it is convenient to set $\mu=2 \cos \theta, 0<\theta<\pi$, and write

$$
\phi_{n}=\alpha \cos (\theta n+\beta)
$$

(d) finally, for $\mu= \pm 2$, we write

$$
\phi_{n}=( \pm 1)^{n}(\alpha n+\beta)
$$

where $\alpha$ and $\beta$ are arbitrary complex constants of integration. The "initial" value of $n$ is not fixed à priori, and by using the freedom to shift $n$, one can remove the constant $\beta$ from the above formulae. Wave functions are bounded in $l^{2}(\mathbf{Z})$ and are not normalizable for the band $|\mu| \leq 2$, which thus represents the continuous spectrum of (3.1). Note that the boundary states with $\mu= \pm 2$ are not doubly degenerate.

There are two well-known classes of orthogonal polynomials that are associated with (3.1). These are the Chebyshev polynomials of the first kind,

$$
T_{n}(\cos \theta)=\cos \theta n, \quad n=0,1, \ldots,
$$

which are the solutions of (3.1) with the boundary conditions $T_{0}=1, T_{1}=\cos \theta$, and the Chebyshev polynomials of the second kind

$$
U_{n}(\cos \theta) \equiv \frac{\sin \theta(n+1)}{\sin \theta}, \quad n=0,1, \ldots,
$$

satisfying the conditions $U_{-1}=0, U_{0}=1$. For both cases, $\mu=2 \cos \theta$. 
Generically, the systems (3.6), (3.7) are infinite dimensional, i.e., the index $n$ goes to infinity. However, there exist families of finite-dimensional polynomials determined by the first zeros of $T_{n}$ and $U_{n}$ which appear when the parameter $\theta$ is a rational part of $\pi$. In these cases, it is convenient to define $p=e^{i \theta}$ and consider primitive roots of unity of the $N$-th degree: $p^{N}=1$. For $N=4 k$, one has $T_{k}=0$, i.e., $k$ is the dimension of a finite set of $T$-polynomials. Simultaneously, one has $U_{2 k-1}=0$, i.e., $2 k-1$ is the dimension of the $U$-polynomials. For $N=4 k+2$, one has $U_{2 k}=0$ and the dimension is equal to $2 k$. Finally, for $N=2 k+1$, one has $U_{2 k}=0$ and the dimension is $2 k$. The spectrum of these roots-of-unity systems is purely discrete, and the corresponding orthogonality relations are defined with respect to discrete measures.

Let us now discuss the impact of a single or (simplified) double Darboux transformation upon the structure of (3.1) and the associated orthogonal polynomials. Using formulae (2.15), (2.16), and (2.17), we obtain for $\phi_{n}=\cosh \omega n$,

$$
\tilde{u}_{n}=\frac{c_{n-1} c_{n+1}}{c_{n}^{2}}, \quad \tilde{b}_{n}=\frac{\sinh ^{2} \omega}{c_{n} c_{n+1}},
$$

where $c_{n} \equiv \cosh \omega n$. The shift $n \rightarrow n-t \sinh \omega / \omega$ in (3.8), where $t$ is a continuous time variable, gives the one-soliton solution of the ordinary Toda lattice [76]. Solutions of the DSE with potentials $\tilde{u}_{n}$ and $\tilde{b}_{n}$ are $\tilde{\psi}_{n}=\psi_{n}-c_{n} \psi_{n+1} / c_{n+1}$. Take $\psi_{n}=e^{\Omega n}$, $\lambda=2 \cosh \Omega, \Omega>0$; then

$$
\tilde{\psi}_{n}=e^{\Omega n}\left(1-e^{\Omega} \frac{\cosh \omega n}{\cosh \omega(n+1)}\right) .
$$

It is seen that for $\omega \neq \Omega$, the function $\tilde{\psi}_{n}$ grows exponentially when $n \rightarrow \infty$. However, for $\omega=\Omega$, the function $\tilde{\psi}_{n} \propto 1 / c_{n+1}$ is normalizable for $n \in \mathbf{Z}$; in the latter case, $\tilde{\psi}_{n}$ corresponds to the only existing bound state in $l^{2}(\mathbf{Z})$.

The complementary characterization of the potentials (3.8) consists of the fact that they describe a subclass of the Askey-Wilson polynomials [6, 7, 28, 58]. Applying Darboux transformations to the Chebyshev polynomials, one gets their explicit form.

If we take the $\mu=2$ state, $\phi_{n}=n+1$, then

$$
\tilde{u}_{n}=\frac{n(n+2)}{(n+1)^{2}}, \quad \tilde{b}_{n}=-\frac{1}{(n+1)(n+2)} .
$$

These potentials correspond to the special class of Jacobi polynomials [25], which also are easily found as Darboux transforms of the Chebyshev polynomials. The DDT with the $\mu \leq-2$ states differs from the cases considered above only by the change of signs of $\tilde{b}_{n}$ and by the corresponding bound state eigenvalue.

A different class of potentials is obtained after application of the double DDT (2.26)-(2.27) to the starting system (3.1)

$$
\tilde{\tilde{u}}_{n}=\frac{c_{n-1} c_{n+2}}{c_{n} c_{n+1}}, \quad \tilde{\tilde{b}}_{n}=0 .
$$

These potentials correspond to the simplest solutions of the Volterra lattice (known also as the Kac-van Moerbeke or Langmiur lattice). They generate again some particular subclasses of the Askey-Wilson polynomials.

Single DDT with the trigonometric wave functions describing continuous spectrum states leads, in general, to potentials $\tilde{u}_{n}$ which are positive on a different interval of $n$ than the original $u_{n}$, and this requires a change of boundary conditions. As shown in the next section, a second Darboux transformation can remove such complications.

Consider the result of applying the Volterra double DDT to the finite-dimensional Chebyshev polynomials of the second kind when $p=e^{i \theta}$ is a particular primitive root 
of unity, $p^{2 N}=1$. For simplicity, we take the lowest root, i.e., we set $\theta=\pi / N$. Then the spectrum of $N-1$ dimensional $U$-polynomials is $\lambda_{l}=2 \cos \pi l / N, l=1, \ldots, N-1$. In this case, the wave function $\phi_{n}=\sin \pi(n+1) / N$ corresponding to the eigenvalue $\mu=2 \cos \pi / N$ leads to

$$
\tilde{\tilde{u}}_{n}=\frac{\sin \pi n / N \sin \pi(n+3) / N}{\sin \pi(n+1) / N \sin \pi(n+2) / N} .
$$

This potential is positive between the closest zeros lying at $n=0$ and $n=N-3$, i.e., it defines a set of polynomials orthogonal with respect to a positive measure. The spectrum of the derived potential (3.10) is $\lambda_{k}=2 \cos \pi k / N, k=2, \ldots, N-2$, i.e., we removed two discrete levels, one from below and one from above. In [72], it was noticed that the potentials (3.10) and their further DDT generalizations define a subset of $q$-ultraspherical polynomials [28]. These polynomials are of finite size, as distinct from the so-called sieved polynomials $[3,11]$. Since they are defined by the periodic potentials, $u_{n+N}=u_{n}$, they are similar to the roots-of-unity systems found in [66]. It is worth mentioning that not all roots of unity have resulting potentials $u_{n}$ that are positive.

Further investigation of the classical orthogonal polynomials that can be derived from dressing (or undressing) of the free DSE is given in Section 8.

\section{Bound states in the continuum}

In this section, we show how singularities generated by the first Darboux transformation may be removed by the second one. In particular, we consider the case when such a double DDT creates a bound state on the background of the continuous spectrum. For the standard Schrödinger equation (1.1), such systems have been systematically considered in [23,37], and for the Jacobi matrices, this procedure was developed in [79].

As was shown in Section 2, the double DDT with the same auxiliary eigenvalue $\tilde{\mu}=\mu$ does not create anything new in the case when the second auxiliary wave function $\tilde{\phi}_{n}$ is a Darboux transform of the wave function with eigenvalue $\mu$. An interesting situation takes place when one performs a second DDT with the general solution of the equation

$$
\tilde{u}_{n+1} \eta_{n+1}+\eta_{n-1}+\tilde{b}_{n} \eta_{n}=\mu \eta_{n}
$$

which is linearly independent from $\tilde{\phi}_{n}$ given by (2.21). A general solution of this DSE looks like

$$
\eta_{n}=\alpha \tilde{\phi}_{n}\left(\beta+\sum_{k=n_{0}}^{n} \frac{\tilde{W}_{k}}{\tilde{\phi}_{k} \tilde{\phi}_{k-1}}\right)
$$

where $\tilde{W}_{n}$ is found from the equation

$$
\frac{\tilde{W}_{n+1}}{\tilde{W}_{n}}=\frac{1}{\tilde{u}_{n+1}}=\frac{W_{n+2}}{W_{n+1}} \frac{\phi_{n+1}^{2}}{\phi_{n+2} \phi_{n}} .
$$

Integrating the latter equation once, we obtain $\tilde{W}_{n}=W_{n+1} \phi_{n} / \phi_{n+1}$, where an arbitrary multiplicative factor is absorbed into the definition of $\tilde{W}_{n}$ since no information about the structure of solutions is lost under such rescaling. Now we rewrite $\eta_{n}$ in the 
form

$$
\eta_{n}=\alpha \frac{y_{n+1} W_{n+1}}{\phi_{n+1}}, \quad y_{n} \equiv \sum_{k=n_{0}}^{n-1} \frac{\phi_{k}^{2}}{W_{k}}+\beta .
$$

This is the general solution for (4.1). The first solution $\tilde{\phi}_{n}$ is recovered in the limit $\alpha \rightarrow 0, \alpha \beta=$ constant.

Consider now how the potentials are transformed under the double DDT performed with the help of the $\phi_{n}$ and $\eta_{n}$ functions

$$
\begin{gathered}
\tilde{u}_{n}=u_{n+2} \frac{W_{n+2} W_{n}}{W_{n+1}^{2}} \frac{y_{n} y_{n+2}}{y_{n+1}^{2}}=u_{n+1} \frac{y_{n} y_{n+2}}{y_{n+1}^{2}}, \\
\tilde{\tilde{b}}_{n}=b_{n}+\frac{\phi_{n-1} \phi_{n}}{W_{n} y_{n+1}}-\frac{\phi_{n} \phi_{n+1}}{W_{n+1} y_{n+2}}+\left(\mu-b_{n}\right) \frac{y_{n}}{y_{n+1}}-\left(\mu-b_{n+1}\right) \frac{y_{n+1}}{y_{n+2}} .
\end{gathered}
$$

These formulae were derived in [79] in a slightly different form. The transformed wave function $\tilde{\tilde{\phi}}_{n}$ is

$$
\tilde{\tilde{\phi}}_{n}=\tilde{\phi}_{n}-\frac{\eta_{n}}{\eta_{n+1}} \tilde{\phi}_{n+1}=\frac{\tilde{W}_{n+1}}{\eta_{n+1}}=\frac{\phi_{n+1}}{y_{n+2}} .
$$

It is interesting to find a criterion when this function belongs to the discrete spectrum of DSE with potentials (4.4), (4.5).

For this, notice that the transformation $\psi_{n}=\sqrt{W_{n}} \chi_{n}$ yields the wave functions $\chi_{n}$, which satisfy DSE in the self-adjoint form (2.3), and the condition of normalizability is

$$
\sum_{n=0}^{\infty} \chi_{n}^{2}=\sum_{n=0}^{\infty} \frac{\psi_{n}^{2}}{W_{n}}<\infty
$$

where we restricted ourselves to the half-line Hilbert space $l^{2}(0, \infty)$. Hence, for the function $\tilde{\tilde{\phi}}_{n}$, we should have the condition

$$
\sum_{n=0}^{\infty}\left(\tilde{\tilde{\phi}}_{n}\right)^{2} / \tilde{\tilde{W}}_{n}<\infty
$$

The Wronskian $\tilde{\tilde{W}}_{n}$ is found from the equation

$$
\tilde{\tilde{W}}_{n+1} / \tilde{\tilde{W}}_{n}=\frac{1}{\tilde{\tilde{u}}_{n+1}}=\frac{y_{n+2}^{2}}{u_{n+2} y_{n+1} y_{n+3}},
$$

i.e., $\tilde{\tilde{W}}_{n}=y_{n+1} W_{n+1} / y_{n+2}$, where the constant of integration has been absorbed into $\tilde{\tilde{W}}_{n}$. To conclude, for $\tilde{\tilde{\phi}}_{n}$ to belong to the discrete spectrum, the following condition must be satisfied

$$
\sum_{n=1}^{\infty} \frac{\phi_{n}^{2}}{W_{n} y_{n} y_{n+1}}<\infty
$$

The double DDT has interesting applications in the theory of orthogonal polynomials. Indeed, consider the system of orthogonal polynomials $P_{n}(x)(x \equiv \lambda)$ satisfying the three-term recurrence relation (2.1) with the initial conditions $P_{-1}=0, P_{0}=1$. 
For $u_{n}>0, b_{n}$ real, these polynomials are orthogonal with respect to some positive weight function $w(x)$

$$
\int_{a}^{b} P_{n}(x) P_{m}(x) w(x) d x=h_{n} \delta_{n m} .
$$

For the first DDT, let us choose $\phi_{n}=P_{n}(\mu)$ where $\mu$ lies to the left of the orthogonality interval $[a, b]$. Then it is well known [17] that the kernel polynomials mentioned above,

$$
\tilde{P}_{n}(x ; \mu)=\frac{1}{x-\mu}\left(P_{n}(x)-\frac{\phi_{n}}{\phi_{n+1}} P_{n+1}(x)\right),
$$

are orthogonal with respect to the weight function

$$
\tilde{w}(x)=(x-\mu) w(x) .
$$

Now we can perform the second DDT with the help of $\eta_{n}$. Consider a system of polynomials defined by the relations

$$
\tilde{\tilde{P}}_{n}(x)=\tilde{P}_{n-1}(x)-\frac{\eta_{n-1}}{\eta_{n}} \tilde{P}_{n}(x)
$$

Proposition 1. For a suitable choice of the constant $\beta$ in (4.3), the polynomials $\tilde{\tilde{P}}_{n}(x)$ are orthogonal with respect to the positive weight function

$$
\tilde{\tilde{w}}(x)=\frac{\tilde{w}(x)}{x-\mu}+J \delta(x-\mu)=w(x)+J \delta(x-\mu),
$$

which differs from the initial $w(x)$ only by the presence of one additional discrete mass $J$ at the point $x=\mu$ (the value of this mass $J$ is related to $\beta$ ).

A major part of this statement goes back to Geronimus [33, 34], who found the weight function for polynomials $\tilde{\tilde{P}}_{n}(x)$ from the given weight function for $\tilde{P}_{n}(x)$. The full statement follows from the assumption that $\tilde{P}_{n}(x)$ are kernel polynomials of $P_{n}(x)$ with the weight function $w(x)$. The double DDT thus yields a simple analytic solution of the problem considered in $[18,82]$, of how to add the discrete mass $J$ outside the spectral interval without changing the weight function. From the last equation, we see that $\tilde{w}(x)=(x-\mu) \tilde{\tilde{w}}(x)$ because $x \delta(x)=0$ (i.e., the mass point is removed). This means that the polynomials $\tilde{P}_{n}(x)$ serve as the kernel polynomials with respect to $\tilde{\tilde{P}}_{n}$ whenever the relations (4.13) hold.

There are interesting situations when the discrete mass is inserted inside the spectral interval $[a, b]$. Indeed, the first DDT (4.11) yields the well-defined orthogonal polynomials provided $\mu<a$ or $\mu>b$; otherwise one fails to have positivity of the weight function $\tilde{w}(x)$. Nevertheless, this DDT is justified if one goes beyond the polynomial systems and considers the resulting DSE as it is. Then the second DDT with $\eta_{n}$ leads to well-defined orthogonal polynomials having discrete mass inside the spectral interval.

Consider the simplest case: the partners of Chebyshev potentials with one bound state inside the continuous spectrum. Since $u_{n}=1, b_{n}=0$, one has $W_{n}=1$. Choose

$$
\phi_{n}=s_{n+1}, \quad s_{n} \equiv \sin \theta n, \quad \mu=2 \cos \theta .
$$

Then from (4.6) we have $\tilde{\tilde{\phi}}_{n}=s_{n+2} / y_{n+2}$ and

$$
\tilde{\tilde{u}}_{n}=\frac{y_{n} y_{n+2}}{y_{n+1}^{2}}
$$




$$
\tilde{\tilde{b}}_{n}=\frac{s_{n} s_{n+1}}{y_{n+1}}-\frac{s_{n+1} s_{n+2}}{y_{n+2}}+\mu\left(\frac{y_{n}}{y_{n+1}}-\frac{y_{n+1}}{y_{n+2}}\right)
$$

where the function $y_{n}$ is found from (4.3) to be

$$
y_{n}=\frac{1}{2}\left(n+\beta-\frac{\sin 2 \theta(n+1 / 2)}{2 \sin \theta}\right) \text {. }
$$

Taking sufficiently large $\beta$, one can assure that $y_{n}>0, n \geq-1$. Then $\tilde{\tilde{u}}_{n}>0$, and the polynomials defined by the recurrence relation

$$
\tilde{\tilde{u}}_{n} \tilde{\tilde{P}}_{n+1}(x)+\tilde{\tilde{P}}_{n-1}(x)+\tilde{\tilde{b}}_{n-1} \tilde{\tilde{P}}_{n}(x)=x \tilde{\tilde{P}}_{n}(x),
$$

with initial conditions $\tilde{\tilde{P}}_{-1}=0, \tilde{\tilde{P}}_{0}=$ constant, are orthogonal with respect to the positive measure with one normalizable state $\tilde{\tilde{P}}_{n}(\mu)$ inside the continuous spectrum

$$
\tilde{\tilde{P}}_{n}(\mu)=\frac{\sin \theta(n+1)}{y_{n+1}} \text {. }
$$

It is clear that $\tilde{\tilde{P}}_{-1}=0$, and the condition (4.9) is fulfilled. When $n \rightarrow \infty$, the potentials oscillate and go to their limiting values $\tilde{\tilde{u}}_{n} \rightarrow 1, \tilde{\tilde{b}}_{n} \rightarrow 0$ very slowly, which is a characteristic feature of potentials with bound states in the continuum.

The above polynomials take especially simple forms when $\theta=\pi / 2$, i.e., when one performs a double DDT with the $\mu=0$ auxiliary level. For this case, we have

$$
y_{n}=\frac{1}{2}\left(n+\beta-\frac{1}{2}(-1)^{n}\right), \quad \tilde{\tilde{u}}_{n}=\frac{y_{n} y_{n+2}}{y_{n+1}^{2}}, \quad \tilde{\tilde{b}}_{n}=0 .
$$

The unique bound state wave function looks as follows:

$$
\tilde{\tilde{P}}_{2 k+1}(0)=0, \quad \tilde{\tilde{P}}_{2 k}(0)=\frac{2(-1)^{k}}{2 k+\beta+3 / 2} .
$$

The recurrence coefficients (4.21) are given in [17, p.112, Ex 2.14], but the polynomials $\tilde{\tilde{P}}_{n}$ differ from the corresponding $S_{n}$ 's by the initial conditions. This example looks similar to the polynomials with discrete masses inside the interval of orthogonality tied to zeros of the Chebyshev polynomials that appeared in the studies of sieved orthogonal polynomials $[13,32,77]$. It would be interesting to find the relationship of the procedure used in these papers to the double DDT.

We conclude that the Darboux transformation technique is a universal instrument that allows us to perform analytically a large variety of spectral "surgeries", namely to add (remove) discrete levels in arbitrary places, to change the masses of the discrete levels, etc. (cf. [84], where a qualitative numerical analysis of similar problems for the discrete Schrödinger equation has been performed). It allows us to construct systematically orthogonal polynomials of the more general type than those of $[13$, 16-18, 32, 39, 47, 77, 82]. In particular, a double DDT allows us to build systems of polynomials with embedded masses at arbitrary points of the interval of orthogonality - a possibility which was not explored systematically in the literature. One also can apply the above techniques to the spectral problem of polynomials orthogonal on the unit circle and get analogous results.

\section{Discrete dressing chain and discrete-time Toda lattice}

There are several versions of the discrete-time analogs of the Toda lattice $[38,42,56$, $73]$. Here we analyze the version which follows from the considerations of $[27,38,53-$ $55,70]$. More precisely, in this and the following sections we derive the corresponding 
DTTL in explicit closed form, discuss its relationship to the discrete dressing chain, and describe various similarity solutions. A systematic approach to discrete lattices determined by the sequences of Darboux (Bäcklund) transformations for differential equations was developed in [65]. For general reviews on Darboux transformations, discrete-time systems, and large lists of references, see $[9,19,80]$. $t$

Consider a tridiagonal Schrödinger operator $H(t)$ depending on a discrete "time"

$$
H(t)|n\rangle=|n+1\rangle+u_{n}(t)|n-1\rangle+b_{n}(t)|n\rangle
$$

(the basis $|n\rangle$ is assumed to be independent of time). We would like to treat the Darboux transformation (1.7) as a time shift, $H(t+h) R(t)=R(t) H(t)$, where $h$ is some positive constant (independent of $t$ ). Let us rewrite the equations (2.6)-(2.8) as

$$
\begin{gathered}
u_{n+1} A_{n}-\tilde{u}_{n} A_{n+1}=0, \\
A_{n}-A_{n+1}=b_{n}-\tilde{b}_{n}, \\
A_{n}\left(\tilde{b}_{n-1}-b_{n}\right)=u_{n}-\tilde{u}_{n},
\end{gathered}
$$

where $\tilde{u}_{n}(t)=u_{n}(t+h), \tilde{b}_{n}(t)=b_{n}(t+h)$. In order to obtain the DTTL, we should exclude $A_{n}$ from this system. From (5.2) and (5.3), one has

$$
A_{n}=\frac{\left(b_{n}-\tilde{b}_{n}\right) \tilde{u}_{n}}{\tilde{u}_{n}-u_{n+1}} .
$$

Substitution of (5.5) into (5.4) results in

$$
\tilde{u}_{n}\left(\tilde{b}_{n}-b_{n}\right)\left(\tilde{b}_{n-1}-b_{n}\right)=\left(\tilde{u}_{n}-u_{n}\right)\left(\tilde{u}_{n}-u_{n+1}\right) .
$$

On the other hand, we can eliminate $A_{n}$ from (5.2) and (5.3),

$$
A_{n+1}=\frac{U_{n+1}\left(b_{n}-\tilde{b}_{n}\right)}{\tilde{u}_{n}-u_{n+1}} \text {. }
$$

Substituting this into (5.4), we derive

$$
u_{n+1}\left(\tilde{b}_{n}-b_{n+1}\right)\left(\tilde{b}_{n}-b_{n}\right)=\left(\tilde{u}_{n}-u_{n+1}\right)\left(\tilde{u}_{n+1}-u_{n+1}\right) \text {. }
$$

Proposition 2. Under the assumption that $u_{n}(t)$ and $b_{n}(t)$ are analytical functions of $t$, the equations (5.6), (5.7), in the limit $h \rightarrow 0$, are reduced to the ordinary Toda lattice equations, i.e., they define a particular form of DTTL.

Proof. Firstly, we note that the analyticity requirement is a strong restriction upon the space of solutions of (5.6), (5.7). Within this subclass of solutions, one may set, for $h \rightarrow 0$,

$$
\tilde{u}_{n} \approx u_{n}+h \dot{u}_{n}, \quad \tilde{b}_{n} \approx b_{n}+h \dot{b}_{n}
$$

Substituting this into (5.6), (5.7), we obtain

$$
\begin{gathered}
u_{n+1}\left(b_{n}-b_{n+1}\right) \dot{b}_{n}=\dot{u}_{n+1}\left(u_{n}-u_{n+1}\right), \\
u_{n}\left(b_{n-1}-b_{n}\right) \dot{b}_{n}=\dot{u}_{n}\left(u_{n}-u_{n+1}\right) .
\end{gathered}
$$

Dividing the first of these equations by the second one, we get

$$
\dot{u}_{n}=f_{1}(t) u_{n}\left(b_{n-1}-b_{n}\right)
$$

where $f_{1}(t)$ is an arbitrary function of time. Analogously, shifting $n \rightarrow n-1$ in (5.9) and dividing by (5.10), we obtain

$$
\dot{b}_{n}=f_{2}(t)\left(u_{n}-u_{n+1}\right) .
$$


The compatibility of (5.11), (5.12) with (5.9), (5.10) yields the constraint $f_{1}(t)=$ $f_{2}(t)$. Then the equations (5.11), (5.12) give the exact Toda lattice equations [76]. The function $f(t)=f_{1,2}(t)$ appears due to the arbitrariness in the continuous time parametrization.

Comment. Equations (5.2)-(5.4) were obtained in [27, 53, 54], but the relationship with DTTL was not noticed. Vice versa, in [38] it was shown that $H(t+h) R(t)=$ $R(t) H(t)$ defines a DTTL, but the explicit local formulae (5.6), (5.7) were not derived. The relationship of the refactorization condition $L(t+h) R(t+h)=R(t) L(t)$ to the DTTL also has been discussed recently in $[43,60,67]$. For a different way of deriving the DTTL, see [78].

The DTTL we have derived can be obtained as the compatibility condition of two linear equations,

$$
\begin{gathered}
\psi_{n}(t+h)=\psi_{n}(t)+A_{n+1}(t) \psi_{n+1}(t), \\
u_{n+1}(t) \psi_{n+1}(t)+\psi_{n-1}(t)+b_{n}(t) \psi_{n}(t)=\lambda \psi_{n}(t),
\end{gathered}
$$

with the additional condition that $A_{n}(t)$ does not depend on the spectral parameter $\lambda$. We remark that the above scheme was analyzed by Ablowitz and Ladik (see [1] and references therein), but the simple ansatz leading to the DTTL (5.6), (5.7) was not considered explicitly.

From the formal relations

$$
H(t+h)=R(t) H(t) R^{-1}(t)=L^{-1}(t) H(t) L(t),
$$

one expects that the operators $H(t)$ form an isospectral family, so that it is possible to construct the integrals of motion such as $\operatorname{Tr} H(t)$, det $H(t)$, and others which do not depend on $t$. However, the equations (5.15) are true provided the operators $R, L$ are well defined and invertible, i.e., if they do not have zero modes,

$$
R(t)|\Phi(t)\rangle_{0}=0, \quad L(t)|\Psi(t)\rangle_{0}=0,
$$

which belong to the physical eigenstates of $H(t)$. Under the natural constraint for the operators $R(t), L(t)$ to have the same domain of definition as $H(t)$, there are three simple possibilities: either $H(t+h)$ has an additional bound state with respect to the Hamiltonian $H(t)$ described by $|\Psi(t)\rangle_{0}$, or it does not have a partner state to $|\Phi(t)\rangle_{0}$, or the spectra of $\tilde{H}$ and $H$ are completely equal.

We see that DTTL (5.6), (5.7) is a necessary but not sufficient condition for the isospectrality: solutions of DTTL provide both strictly isospectral and almost isospectral Hamiltonians. In the latter case, the number and the explicit form of the missing states are controlled by the equations (5.16). Taking into account that $A_{n}(t)=-\phi_{n-1} / \phi_{n}$ where $\phi_{n}$ is a solution of (2.14) with an eigenvalue $\mu$, we see that the sufficient conditions for DTTL to have isospectral solutions a.t each discrete time value are: (a) $\mu(t)$ should lie outside of the spectrum of $H(t)$; (b) $A_{n}(t)$ should not contain singularities; (c) zero modes of the reciprocal DDT operator $L(t)$ should not satisfy given boundary conditions (like normalizability, $\psi_{-1}=0$, etc.).

The wave function $\psi_{n}(t+h)$ is the Darboux transform of $\psi_{n}(t)$. The limit $h \rightarrow 0$ corresponds to the "infinitesimal" Darboux transformation. This limit means that $A_{n}$ is sufficiently small, i.e., $\mu$ is large. One can parametrize (up to terms $\propto h^{2}$ ) $\mu=1 / h$, $A_{n}=h x_{n}\left(1+h y_{n}\right)$. Substituting this ansatz into (2.12), we obtain

$$
A_{n}=-h u_{n}\left(1+h b_{n-1}\right) \text {. }
$$


Note that this approximation is nothing other than the WKB approximation for DSE (2.14) (i.e., the large $\mu$ asymptotics). We see that, as in standard quantum mechanics, the Riccati representation of DSE provides a simple analysis of the WKB limit.

Let us derive a discrete-time analog of the Volterra lattice. Consider the system of equations

$$
\begin{gathered}
\psi_{n}(t+h)=\psi_{n}(t)+D_{n+2}(t) \psi_{n+2}(t) \\
u_{n+1}(t) \psi_{n+1}(t)+\psi_{n-1}(t)=\lambda \psi_{n}(t)
\end{gathered}
$$

The compatibility conditions give

$$
\tilde{u}_{n}=u_{n+2} D_{n+1} / D_{n+2}, \quad \tilde{u}_{n}+D_{n}=u_{n}+D_{n+1},
$$

where $\tilde{u}_{n}(t)=u_{n}(t+h)$. Eliminating the variables $D_{n}$, we get the discrete-time Volterra lattice

$$
u_{n+1}\left(\tilde{u}_{n}-u_{n}\right)\left(\tilde{u}_{n}-u_{n+2}\right)=\tilde{u}_{n}\left(\tilde{u}_{n-1}-u_{n+1}\right)\left(\tilde{u}_{n+1}-u_{n+1}\right) .
$$

For $h \rightarrow 0$, one obtains the standard Volterra chain equations

$$
\dot{u}_{n}=f(t) u_{n}\left(u_{n+1}-u_{n-1}\right),
$$

where $f(t)$ is an arbitrary continuous function of time.

Analogous to the Toda case, one can integrate equations (5.19) and get the quadratic Riccati equation

$$
\left(D_{n}-u_{n}\right)\left(D_{n+1}-u_{n+1}\right)=-\mu^{2} D_{n+1} .
$$

Substituting the ansatz $D_{n}=-\phi_{n-2} / \phi_{n}$ and integrating the resulting equation once, we get the DSE

$$
u_{n+1} \phi_{n+1}+\phi_{n-1}=\mu\left(\sqrt{1+\alpha^{2}}+\alpha(-1)^{n}\right) \phi_{n}
$$

where $\alpha$ is another integration constant. For $\alpha=0$, this is the standard two-diagonal DSE. But for $\alpha \neq 0$, it is necessary to split even and odd lattice points, and then the wave functions $\phi_{2 k}$ and $\phi_{2 k+1}$ satisfy certain tridiagonal DSEs. In the WKB approximation, we set $\mu^{2}=h^{-1}, h \rightarrow 0$, and expand all variables in (5.22) up to terms $\propto h^{2}$. This gives

$$
D_{n}=-h u_{n} u_{n-1}\left(1-h\left(u_{n-1}+u_{n-2}\right)\right),
$$

and the relations (5.19) are reduced to the Volterra lattice: $\dot{u}_{n}=u_{n}\left(u_{n+1}-u_{n-1}\right)$.

For the sake of completeness, we now discuss briefly the differential Schrödinger equation. It is not difficult to derive an analog of the DTTL resulting from the intertwining relations (1.7) for the differential operator $H$ (1.1). Using the standard formulae for the factorization method [44], we get the nonlinear differential-difference equation

$$
\left(\tilde{u}^{\prime \prime}+u^{\prime \prime}\right)(\tilde{u}-u)+\left(u^{\prime}\right)^{2}-\left(\tilde{u}^{\prime}\right)^{2}=(\tilde{u}-u)^{3}
$$

where $u=u(x, t), \tilde{u}=u(x, t+h), u^{\prime}=d u / d x$, and $x$ is the continuous space coordinate. In the limit $h \rightarrow 0$, one can substitute $\tilde{u} \approx u+h d u / d t$ into (5.25) and find its continuous time partner to be $d u / d x=f(t) d u / d t$ where $f(t)$ is an arbitrary function of time.

Let us rewrite (1.6) using the time-dependence notations

$$
\begin{gathered}
L(t+h) R(t+h)=H(t+h)-\mu(t+h), \\
R(t) L(t)=H(t+h)-\mu(t) .
\end{gathered}
$$


Excluding the operator $H$ from these relations, we have

$$
L(t+h) R(t+h)=R(t) L(t)+\mu(t)-\mu(t+h),
$$

which is nothing other than the refactorization condition $[44,55]$. The components of $(5.26),(5.27)$ are

$$
\begin{gathered}
u_{n}(t)=A_{n}(t) C_{n-1}(t), \\
b_{n}(t)=A_{n}(t)+C_{n}(t)+\mu(t), \\
A_{n}(t+h) C_{n-1}(t+h)=A_{n}(t) C_{n}(t), \\
A_{n}(t+h)+C_{n}(t+h)=A_{n+1}(t)+C_{n}(t)+\mu(t)-\mu(t+h) .
\end{gathered}
$$

The latter two equations define the discrete dressing chain $[27,55,70]$. The functions $A_{n}$ and $C_{n}$ play the role of superpotentials within the supersymmetric interpretation of (5.28), (5.29). They obey the discrete Riccati equations

$$
A_{n}+u_{n+1} / A_{n+1}-b_{n}=-\mu, \quad C_{n}+u_{n} / C_{n-1}-b_{n}=-\mu,
$$

and can be expressed through solutions of the DSE (2.14).

It is evident that any solution of $(5.30),(5.31)$ provides a solution of the DTTL. The inverse statement is also true. For any solution $u_{n}(t), b_{n}(t)$ of the DTTL, the functions $A_{n}(t), C_{n}(t), \mu(t)$ are unique:

$$
\begin{gathered}
A_{n}(t)=u_{n}(t+h) \frac{b_{n}(t)-b_{n}(t+h)}{u_{n}(t+h)-u_{n+1}(t)}, \\
C_{n}(t)=\frac{u_{n+1}(t)}{A_{n+1}(t)}, \quad \mu(t)=b_{n}(t)-A_{n}(t)-C_{n}(t) .
\end{gathered}
$$

We conclude that the DTTL and the discrete dressing chain are completely equivalent to each other. In fact, the discrete dressing chain is the first integral of the DTTL (5.6), (5.7), which follows from the considerations of Section 2 where the parameter $\mu(t)$ has been introduced. Indeed, substituting the expansion (5.17) into the above formulae yields the Toda equations. Thus, the discrete dressing chain may be interpreted as the discrete-time Toda lattice.

Suppose that we have potentials $u_{n}, b_{n}$ for which the DSE (2.1) is exactly solvable, and let the spectrum be purely discrete. Since we know both the eigenvalues $\lambda_{k}$, $k=1,2, \ldots$, and the corresponding wave functions $\psi_{n}^{(k)}$, we can build the discrete dressing chain just by taking $\mu(1)=\lambda_{1}, \phi_{n}(1)=\psi_{n}^{(1)}, \mu(2)=\lambda_{2}, \phi_{n}(2)=R(1) \psi_{n}^{(2)}$, and so on. This corresponds to the consecutive removal of all discrete levels from the spectrum of the initial Hamiltonian $H$. This simple observation shows that any solvable DSE is related to some solution of the discrete dressing chain such that $\lambda_{n}$ describes the corresponding discrete spectrum. Otherwise, any solvable DSE defines an infinite number of solutions of the DTTL, which are analytical in discrete time $j$ only in very special cases. The dressing technique is useful for the search and classification of exactly solvable problems because it allows us to work with entire classes of systems obeying certain fixed symmetry properties. In the next section, we show that the similarity solutions of the DTTL (solutions with additional symmetries) lead to DSE's determining many classical special functions.

For the Volterra chain, we have the following ansatz:

$$
\begin{gathered}
H|n\rangle=|n+1\rangle+u_{n}|n-1\rangle, \\
R|n\rangle=|n\rangle+A_{n}|n-2\rangle, \quad L|n\rangle=C_{n}|n\rangle+|n+2\rangle,
\end{gathered}
$$


where, for simplicity, we use the same notations $R, L$, and $A_{n}, C_{n}$ for the DDT operators and superpotentials ( $A_{n}$ is equal to the $D_{n}$ above). Intertwining relations $\tilde{H} R=R H, L \tilde{H}=H L$ give

$$
\begin{gathered}
C_{n}=u_{n+1} u_{n+2} / A_{n+2}, \quad A_{n} \tilde{u}_{n-2}=u_{n} A_{n-1}, \\
\left(u_{n}-A_{n}\right)\left(u_{n+1}-A_{n+1}\right)=-\mu^{2} A_{n+1},
\end{gathered}
$$

where $\mu$ is an eigenvalue of the DSE (5.23).

From the factorization conditions and equations (5.34), (5.35), it follows that

$$
L R=H^{2}-\mu^{2}, \quad R L=\tilde{H}^{2}-\mu^{2} .
$$

One also can introduce the time variable $t$ such that $\tilde{L} \tilde{R}+\tilde{\mu}^{2}=R L+\mu^{2}$ where $\tilde{L} \equiv L(t+h)$, etc. In components, one has

$$
\begin{gathered}
\tilde{A}_{n} \tilde{C}_{n-2}=A_{n} C_{n}, \\
\tilde{A}_{n}+\tilde{C}_{n}+\tilde{\mu}^{2}=A_{n+2}+C_{n}+\mu^{2} .
\end{gathered}
$$

These equations are the descendants of the discrete dressing chain (5.30), (5.31). Once the superpotentials $A_{n}$ and $C_{n}$ are known, one can find the potential $u_{n}$ from the quadratic equation

$$
u_{n}^{2}-u_{n}\left(A_{n}+C_{n}+\mu^{2}\right)+A_{n+1} C_{n-1}=0 .
$$

Proposition 3. The $A_{n}$ and $C_{n}$ solutions of the discrete dressing chain (5.30), (5.31), which are analytical in $n$, can be split into two classes with the help of a (multivalued) decomposition

$$
\begin{gathered}
A_{n}(j)=V_{2 n}(j) V_{2 n+1}(j), \\
C_{n}(j)=\left(\nu(j)-V_{2 n+1}(j)\right)\left(\nu(j)-V_{2 n+2}(j)\right), \\
\mu(j)=\text { constant }-\nu^{2}(j) .
\end{gathered}
$$

In the first case, $V_{n}(j)$ satisfies the second order in $n$ and first order in $j$ differencedifference equation

$$
V_{n}(j+1)\left(\nu(j+1)-V_{n-1}(j+1)\right)=V_{n}(j)\left(\nu(j)-V_{n+1}(j)\right),
$$

which is a new form of the discrete-time Volterra lattice. In the second, rather trivial case, $V_{n}(j)$ satisfies the equation

$$
V_{n}(j+1)\left(\nu(j+1)-V_{n-1}(j+1)\right)=V_{n-1}(j)\left(\nu(j)-V_{n}(j)\right)
$$

and the additional constraint

$$
V_{n-1}(j)\left(\nu(j)-V_{n}(j)\right)=V_{n+1}(j)\left(\nu(j)-V_{n+2}(j)\right)
$$

This statement is proved by direct substitution of the ansatz (5.39) into the original dressing chain (5.30), (5.31) and simple algebraic manipulations. In the following, we neglect the second possibility (5.41). In order to see that (5.40) defines a discrete-time Volterra lattice, it is sufficient to consider the limit $h \rightarrow 0, \nu=1 / h$, and use the expansion $V_{n}(t+h) \approx u_{n}(t)+h \dot{u}_{n}(t)$. Note that the function $u_{n}(j)=V_{n}(j)\left(\nu(j)-V_{n-1}(j)\right)$ satisfies the original discrete-time Volterra lattice (5.20). Since, in this picture, we have a factorization of the superpotentials $A_{n}, C_{n}$, we call the chain (5.40) the second factorization chain. The above formulae can be interpreted as a discrete-time analog of the well-known connection between solutions of the Volterra and Toda equations (for example, see [35]). The main advantage of representation (5.39), (5.40) consists in the replacement of two nonlinear equations of the discrete dressing chain by one equation in a much simpler form. To the authors' knowledge, the equation (5.40) is new and, in 
light of a large number of applications of the discrete dressing procedure, it deserves careful investigation.

\section{Similarity reductions and special solutions of the DTTL}

We have seen that the DTTL can describe isospectral time evolution of the potentials of DSE. Therefore, one can expect special solutions such as ordinary wave solutions, solitons, etc., to appear after various similarity reductions (as was the case for the ordinary Toda lattice $[51,76,87])$. The most interesting feature of similarity solutions of dressing chains is the presence of a large number of symmetries, so that the corresponding linear problems are expected to incorporate at least the classical special functions.

Let us consider some simple similarity reductions of the DTTL. First of all, consider the running wave solutions.

(i) If one imposes the ansatz $u_{n}(t)=u(t+n h), b_{n}(t)=b(t+n h)$, then the equations (5.6), (5.7) are fulfilled identically. Therefore, any $u_{n}(t)$ and $b_{n}(t)$ describing waves that run to the left of the lattice with equal velocities provide a solution of the DTTL.

(ii) If one sets $u_{n}(t)=u(t-n h), b_{n}(t)=b(t-n h)$, then we obtain nontrivial solutions expressed in terms of elliptic functions. This can be found directly from the equations (5.6), (5.7), but it is instructive to consider the algebraic approach as well. The suggested ansatz means that $\tilde{u}_{n}=u_{n-1}, \tilde{b}_{n}=b_{n-1}$, so that the operator $\tilde{H}=H(t+h)$ can be written in the form

$$
H(t+h)=T H(t) T^{-1}
$$

where $T$ is the shift operator, $T^{ \pm 1}|n\rangle=|n \pm 1\rangle$. Then the Darboux transformation can be represented as the operator identity

$$
T H T^{-1} R=R H, \quad \text { or } \quad\left[H, R^{\prime}\right]=0
$$

where $R^{\prime}=T^{-1} R$. We conclude that all wave type solutions running to the right of the lattice can be derived from the condition (6.2) expressing the commutativity of two operators, $H$ and $R^{\prime}$.

Relations (6.2) are equivalent to the equations

$$
\begin{gathered}
A_{n} u_{n-2}=u_{n} A_{n-1}, \\
A_{n} b_{n-2}+u_{n-1}=u_{n}+b_{n} A_{n}, \\
A_{n}+b_{n-1}=A_{n+1}+b_{n} .
\end{gathered}
$$

They can easily be integrated to the form

$$
u_{n}^{2} u_{n+1}^{2}+\alpha u_{n} u_{n+1}+\beta\left(u_{n}+u_{n+1}\right)=\gamma
$$

where $\alpha, \beta, \gamma$ are arbitrary constants. It is well known that the biquadratic recurrence relations like (6.4) can be solved in terms of the elliptic functions [8,61].

Note that the wave-type reduction in the more general form

$$
u_{n}(t)=u(k t-n h), \quad b_{n}(t)=b(k t-n h), \quad k=0, \pm 1, \pm 2, \ldots,
$$

leads to the commutativity condition

$$
\begin{gathered}
{\left[H, R_{k}\right]=0, \quad R_{k}=T^{-k} R,} \\
R_{k}|n\rangle=|n-k\rangle+A_{n}|n-k-1\rangle .
\end{gathered}
$$


These solutions are expressed through the hyperelliptic functions due to the observation by Krichever [49] that all commutative difference operators of finite order lead to such functions.

(iii) Somewhat more complicated reductions are

$$
u_{n}(t)=u(k t-n h), \quad b_{n}(t)=b(k t-n h)+\beta n,
$$

where $\beta$ is a constant. For them, we have

$$
H(t+h)=T^{k} H(t) T^{-k}+k \beta ;
$$

hence the intertwining $\tilde{H} R=R H$ is equivalent to the commutator relation

$$
\left[H, R_{k}\right]=-\beta k R_{k} .
$$

This is the spectrum generating algebra ensuring that the spectrum of $H$ is linear. Some of the potentials fixed by the relation (6.9) correspond to the specific classical orthogonal polynomials of a discrete variable, but in general, solutions of (6.9) are related to the discrete Painlevé transcendents [70, 71].

Note that the non-singular running-wave solutions correspond to completely isospectral deformations because the potentials from the family (6.5) differ from each other only by a shift of the argument. For reduction (6.7), the spectrum of $H(t)$ changes with time, but the discrete-time evolution of the operator $H(t)-k \beta t / h$ is isospectral.

(iv) The constraints $u_{n}(t+N h)=q^{2} u_{n-k}(t), b_{n}(t+N h)=q b_{n-k}(t)$ correspond to the $q$-deformation of the algebraic relation (6.9),

$$
M H=q H M, \quad M=T^{-k} R(t+(N-1) h) \cdots R(t+h) R(t) .
$$

This class of closures was found in [70], and realization of the algebra (6.10) for the Schrödinger equation (1.1) has been described earlier in [69].

(v) One also can use the ansatz with separation of spatial and time variables $u_{n}(t)=$ $u(t) \alpha_{n}$. In the case of an ordinary Toda lattice, this leads to a large set of classical orthogonal polynomials (Meixner, Krawtchouk, Charlier, Pollaczek, Laguerre, and Hermite) associated with the standard Lie algebras [87].

(vi) A combination of the running wave and the separated form

$$
u_{n}(t)=u(t+n h) \alpha_{n},
$$

leads to solutions of the DTTL being related to a variety of classical $q$-orthogonal polynomials (dual $q$-Hahn polynomials). Algebraically, it corresponds to the specific quantum algebras (for example see [72]). Note that the reductions similar to (i)-(vi) may be applied to the differential-difference equation (5.25) as well (cf. [64, 68, 69]).

Proposition 4. A generalized separation of variables in the second factorization chain (5.40) determined by the ansatz

$$
V_{2 n}(t)=\frac{\phi(t) r_{n}}{g(2 n h+t)}, \quad V_{2 n+1}(t)=\frac{\sigma(t) p_{n}}{g(2 n h+t+h)},
$$

is integrable in terms of elementary functions. It yields recurrence coefficients for two large classes of orthogonal polynomials: the (associated) Askey-Wilson polynomials and the q-generalizations of the Carlitz-Karlin-McGregor polynomials investigated by Askey and Ismail [5].

The detailed proof of this proposition lies beyond the scope of the present work.

The similarity reductions presented above do not exhaust all possible cases. In order to make a full list of them, one needs to find all continuous and discrete symmetries of the DTTL (or of the discrete dressing chain), to classify all subgroups of symmetries, 
and to look for solutions invariant under these subgroups. Since, at present, there is no complete theory of symmetries for the finite-difference equations, such a program is difficult to perform.

\section{Isospectral families of the Meixner polynomials}

In this section, we give a group-theoretical interpretation of the discrete-time evolution on the example of Meixner polynomials. Consider the generator $K_{0}$ in the representation of positive discrete series $D_{j}^{+}$of the $\mathrm{su}(1,1)$ algebra

$$
K_{0}|n\rangle=|n+1\rangle+\frac{1}{4} \sinh ^{2} \theta n(n+2 j-1)|n-1\rangle+\cosh \theta(n+j)|n\rangle
$$

where $n=0,1, \ldots, \infty$. The spectrum of this operator is $j, j+1, j+2, \ldots$ Let us introduce the "time" $t=2 j h$, and the Hamiltonian $H(t)=K_{0}-j$. Obviously, the spectrum of $H(t)$ is $0,1,2, \ldots$, and it does not depend on $t$, i.e., the operators $H(t)$ form an isospectral family. Note that this system is a representative of the reduction (6.11) where $\alpha_{n}=n \sinh ^{2} \theta / 4 h, u(t)=t-h$. The eigenvalue problem for the operator $H(t)$ generates the Meixner polynomials [26, 40, 52, 58]

$$
\frac{1}{4} \sinh ^{2} \theta(n+1)\left(n+\frac{t}{h}\right) M_{n+1}+M_{n-1}+\left(\cosh \theta\left(n+\frac{t}{2 h}\right)-\frac{t}{2 h}\right) M_{n}=x M_{n}
$$

where $M_{n}=M_{n}(x ; t, \theta), M_{0}=1, M_{-1}=0$, and the spectral parameter $x=0,1, \ldots$ is the argument of the polynomials.

It is easy to verify that the (re)factorization equations (5.28)-(5.31) are satisfied if

$$
A_{n}=n \sinh ^{2} \frac{\theta}{2}, \quad C_{n}=\left(n+\frac{t}{h}\right) \cosh ^{2} \frac{\theta}{2}, \quad \mu(t)=-\frac{t}{h} .
$$

It can be checked that the DDT auxiliary wave function $\phi_{n}(t), A_{n}=-\phi_{n-1} / \phi_{n}$, has the eigenvalue $\mu(t)<0$ and is not normalizable. Zero modes of the reciprocal DDT operator $L(t)$ do not satisfy boundary condition $\psi_{-1}=0$. As a result, the time evolution is isospectral: we neither introduce new levels nor remove the existing ones. Therefore, the Darboux transformations can be rewritten as some identities for the Meixner polynomials [17]:

$$
M_{n}(x ; t+1, \theta)=F(x ; t, \theta)\left(M_{n}(x ; t, \theta)+\sinh ^{2} \frac{\theta}{2}(n+1) M_{n+1}(x ; t, \theta)\right) .
$$

(Recall that the Darboux operator can be multiplied by an arbitrary function $F(x ; t, \theta)$ without changing the basic relation (1.7).) The function $F(x ; t, \theta)$ is fixed from the relation

$$
M_{1}(x ; t, \theta)=\frac{4\left(x h-t \sinh ^{2} \theta / 2\right)}{t \sinh ^{2} \theta} .
$$

Setting $n=0$ in (7.4) and using (7.5), we find that $F(x ; t, \theta)=t \cosh ^{2} \frac{\theta}{2} /(x h+t)$.

Consider the limit $h \rightarrow 0, \theta \rightarrow 0, \theta^{2} / 4 h \rightarrow 1$. Then on the one hand, the recurrence relation (7.2) is reduced to that for the Charlier polynomials,

$$
t(n+1) C_{n+1}(x ; t)+C_{n-1}(x ; t)+(n+t) C_{n}(x ; t)=x C_{n}(x ; t),
$$

$x=0,1, \ldots$ On the other hand, we know that the $h \rightarrow 0$ limit corresponds to the ordinary Toda lattice and separation of variables $u_{n}(t)=u(t) \alpha_{n}$. One can verify that the recurrence coefficients for the Charlier polynomials (7.6) define a solution of the Toda equations [87]. Note that the limit $h \rightarrow 0$ implies $j \rightarrow \infty$ as seen from 
the relation $t=2 j h$, so that we obtain a contraction of the $\operatorname{su}(1,1)$ algebra into the Heisenberg-Weyl one.

The key observation of this section is that the "time" $t$ corresponds to the Casimir parameter $j$ of the $\mathrm{su}(1,1)$ algebra representation. Therefore, the evolution in discretetime is equivalent to passing from one irreducible representation to another. This can be contrasted with the case of continuous time Toda equations where the evolution is equivalent to changing $\theta$, whereas the Casimir parameter $j$ is fixed [87].

\section{Dressing and the Askey-Wilson polynomials}

Consider the three-term recurrence relation for the Askey-Wilson polynomials [6, 7]

$$
u_{n+1} P_{n+1}+P_{n-1}+b_{n} P_{n}=2 x P_{n}, \quad P_{-1}(x) \equiv 0, \quad P_{0}(x) \equiv 1,
$$

where

$$
\begin{gathered}
u_{n}=\frac{\left(1-q^{n}\right)\left(1-g q^{n-2}\right) \prod_{i<k}^{4}\left(1-a_{i} a_{k} q^{n-1}\right)}{\left(1-g q^{2 n-3}\right)\left(1-g q^{2 n-2}\right)^{2}\left(1-g q^{2 n-1}\right)} \\
b_{n}=\frac{q^{n-1}\left[\left(1+g q^{2 n-1}\right)\left(s q+s^{\prime} g\right)-q^{n-1}(1+q) g\left(s+s^{\prime} q\right)\right]}{\left(1-g q^{2 n-2}\right)\left(1-g q^{2 n}\right)} \\
g=a_{1} a_{2} a_{3} a_{4}, \quad s=\sum_{i=1}^{4} a_{i}, \quad s^{\prime}=\sum_{i=1}^{4} a_{i}^{-1}
\end{gathered}
$$

The explicit form of $P_{n}(x)$ is

$$
P_{n}\left(x ; a_{i}\right)=h_{n 4} \varphi_{3}\left(\begin{array}{c}
q^{-n}, g q^{n-1}, a_{1} e^{i \theta}, a_{1} e^{-i \theta} \\
a_{1} a_{2}, a_{1} a_{3}, a_{1} a_{4}
\end{array} ; q, q\right)
$$

where $x=\cos \theta$ and

$$
\begin{gathered}
h_{n}=\frac{a_{1}^{-n}\left(g ; q^{2}\right)_{n}\left(g q ; q^{2}\right)_{n}}{(q ; q)_{n}\left(a_{2} a_{3} ; q\right)_{n}\left(a_{2} a_{4} ; q\right)_{n}\left(a_{3} a_{4} ; q\right)_{n}}, \\
(a ; q)_{n}=(1-a)(1-a q) \cdots\left(1-a q^{n-1}\right) .
\end{gathered}
$$

For completeness, we present the definition of a general basic hypergeometric series according to [28],

$$
{ }_{r} \varphi_{s}\left(\begin{array}{l}
f_{1}, f_{2}, \ldots, f_{r} \\
d_{1}, d_{2}, \ldots, d_{s}
\end{array} ; q, z\right)=\sum_{n=0}^{\infty} \frac{\left(f_{1} ; q\right)_{n}\left(f_{2} ; q\right)_{n} \cdots\left(f_{r} ; q\right)_{n}}{(q ; q)_{n}\left(d_{1} ; q\right)_{n} \cdots\left(d_{s} ; q\right)_{n}}\left[(-1)^{n} q^{n(n-1) / 2}\right]^{1+s-r} z^{n}
$$

where $r, s$ are arbitrary positive integers, and $f_{1}, \ldots, f_{r}, d_{1}, \ldots, d_{s}$ are free parameters. If the parameters $a_{i}$ and $q$ in (8.4) are real, $-1<q<1$, and $\left|a_{i}\right|<1$, then the AskeyWilson polynomials are orthogonal with respect to a continuous weight function on the interval $-1<x<1$. If the absolute value of at least one of the parameters is larger than 1 , then there exists a discrete spectrum in addition to the continuous part (for some exceptions, see $[41,46]$ ).

The Askey-Wilson potentials (8.2), (8.3) provide at least four types (by the number of parameters) of analytical solutions of the discrete dressing chain or DTTL. Let us assume that one of the polynomials' parameters, say $a_{1}$, depends exponentially on time, $a_{1}(t)=a_{1} q^{t}$ (we normalize the time step $h=1$ ). Then we have the following 
solution of the (re)factorization conditions (5.28)-(5.31)

$$
\begin{gathered}
A_{n}(t)=-\frac{a_{1}(t)\left(1-q^{n}\right)\left(1-a_{2} a_{3} q^{n-1}\right)\left(1-a_{2} a_{4} q^{n-1}\right)\left(1-a_{3} a_{4} q^{n-1}\right)}{\left(1-g(t) q^{2 n-2}\right)\left(1-g(t) q^{2 n-1}\right)} \\
C_{n}(t)=-\frac{a_{1}^{-1}(t)\left(1-g(t) q^{n-1}\right)\left(1-a_{1}(t) a_{2} q^{n}\right)\left(1-a_{1}(t) a_{3} q^{n}\right)\left(1-a_{1}(t) a_{4} q^{n}\right)}{\left(1-g(t) q^{2 n-1}\right)\left(1-g(t) q^{2 n}\right)}, \\
\mu(t)=a_{1}(t)+a_{1}^{-1}(t) .
\end{gathered}
$$

Let us take $\left|a_{1}\right|>1$ and $\left|a_{i}\right|<1, i=2,3,4$. Then, from the theory of generic Askey-Wilson polynomials [7], it is known that the number of discrete levels $N$ is determined by the maximal power of $q$ allowed in the inequality $1<a_{1} q^{N-1}$. One step of the above DDT yields $t \rightarrow t+1$, or $a_{1} \rightarrow a_{1} q$, which means that the number of discrete levels is reduced by one. So the taken wisp of Darboux transformations provides an isospectral family of the Askey-Wilson polynomials only when $\left|a_{1}\right|<1$. From the general properties of the factorization method, it follows that $2 x_{k}=\mu(k)$ given by (8.8) describe the discrete spectrum of the system, provided the auxiliary eigenfunctions $\phi_{n}(k)$ are normalizable, in which case they coincide with the boundstate wave functions.

It is convenient to use the parametrization

$$
a_{i} \equiv q^{\alpha_{i}}, \quad g \equiv q^{2 \gamma}, \quad 2 \gamma=\alpha_{1}+\alpha_{2}+\alpha_{3}+\alpha_{4},
$$

and the following compact representation of potentials (8.2), (8.3)

$$
\begin{gathered}
u_{n+1-\gamma}=2^{4} \frac{s_{n+\gamma-1} s_{n-\gamma+1}}{s_{2 n}^{2} s_{2 n-1} s_{2 n+1}} \prod_{i<k} s_{n+\alpha_{i}+\alpha_{k}-\gamma}, \\
b_{n+1-\gamma}=\sigma \sum_{k=1}^{4} \frac{c_{2 n+1} c_{2 \alpha_{k}-2 \gamma+1}-c_{1} c_{2 \alpha_{k}-1}}{s_{2 n} s_{2 n+2}},
\end{gathered}
$$

where the symbols may have either hyperbolic or trigonometric meaning. For the hyperbolic notation, one has

$$
q \equiv e^{-2 \omega}, \quad s_{n} \equiv \sinh \omega n, \quad c_{n} \equiv \cosh \omega n, \quad \sigma=1 .
$$

For the trigonometric notation, one has

$$
q \equiv e^{-2 i \varphi}, \quad s_{n} \equiv \sin \varphi n, \quad c_{n} \equiv \cos \varphi n, \quad \sigma=-1 .
$$

The two representations are connected to each other by the relation $\omega \equiv i \varphi$.

Suppose that the parameters $\alpha_{j}$ (and so $\gamma$ ) are real. Then it is easy to see that the potentials $u_{n}, b_{n}$ are real for real $q, 0 \leq|q| \leq 1$, or for $q=e^{-2 i \varphi}, \varphi$ real. In the hyperbolic case, one has $u_{n}>0$ whenever real $c_{n}$ lie to the right of all zeros and poles in (8.9), and this leads to the polynomials of unlimited degree. In the trigonometric case, $c_{n}, s_{n}$ belong to the interval $[-1,1]$, and in general $u_{n}$ takes negative values. The $q$-Racah polynomials characterized by the quantization condition $a_{i} a_{k}=q^{-N}$ where $N$ is a positive integer and $a_{i}, a_{k}$ are two fixed parameters, were investigated in [6]. They are defined both for real $q,-1<q<1$, and complex $q,|q|=1$. The example (3.10) belongs to a different, new class of finite-dimensional polynomials orthogonal with respect to a positive measure, because zeros of $u_{n}=0$ appear from the different quantization conditions $g=a_{1} a_{2} a_{3} a_{4}=q^{M}, q^{N}=1$. Due to the finite-dimensionality and discreteness of the measure, it also is appropriate to identify these polynomials as $q$-Racah polynomials. From (8.2), one can see that other types of zeros of $u_{n}$ arise when $g=q^{-N}$ for arbitrary $q$, for $q^{N}=1$ and generic values of parameters, and when 
$q^{N}=1, a_{i} a_{k}=q^{M}$. As far as the authors understand, no complete classification of the regions of positive measure for the complex parameters $a_{i}$ and $q$ for the Askey-Wilson potentials has been given. Later we hope to discuss this question in detail.

Proposition 5 ([7]). The potentials (8.2), (8.3) are reduced to the Chebyshev polynomial potentials $u_{n}=1, b_{n}=0$ (free DSE case) for all values of $n$ if and only if the parameters $a_{i}$ are different from each other and take the values

$$
a_{i}=\left(q,-q, q^{1 / 2},-q^{1 / 2}\right), \quad g \equiv q^{3},
$$

or

$$
a_{i}=\left( \pm 1, \mp q, q^{1 / 2},-q^{1 / 2}\right), \quad g \equiv q^{2},
$$

or

$$
a_{i}=\left(1,-1, q^{1 / 2},-q^{1 / 2}\right), \quad g \equiv q,
$$

in arbitrary combination.

It is natural to define potentials to be Darboux equivalent if they can be converted into each other by a number of Darboux transformations. For an infinite number of transformations, this definition should be applied carefully since the existence questions become delicate. From Proposition 5 one can easily deduce Proposition 6 .

Proposition 6. The general subset of the Askey-Wilson potentials, which are Darboux equivalent to $u_{n}=1, b_{n}=0$, is fixed by the following choice of parameters (in arbitrary combination)

$$
a_{i}=\left(q^{n_{1}},-q^{n_{2}}, q^{n_{3}+1 / 2},-q^{n_{4}+1 / 2}\right), \quad a_{i} \neq a_{k} \text { for } i \neq k,
$$

where $n_{1}, n_{2}, n_{3}, n_{4}$ are arbitrary integers.

Indeed, let Chebyshev potentials $u_{n}=1, b_{n}=0$ be fixed by the choice $a_{1}=$ $-a_{2}=q, a_{3}=-a_{4}=q^{1 / 2}$. The DDT (8.6), (8.8) allows us to change $a_{1}=q$ to $a_{1}=q^{n_{1}}, n_{1}>0$ after the $n_{1}-1$ steps. The negative discrete-time evolution, $t \rightarrow t-1$, is governed by the reciprocal DDT operator $L(t)$, which allows us to get $a_{1}=q^{n_{1}}, n_{1} \leq 0$. Other wisps of DDT performing the maps $a_{i} \rightarrow a_{i} q, i=2,3,4$, are obtained from the above formulae just by permutation of the parameters, since the latter entered the potentials (8.2), (8.3) in a symmetric fashion. Because all three sets of parameters leading to free DSE (8.11)-(8.13) are contained in the conditions (8.14), they characterize all Askey-Wilson potentials which are Darboux equivalent to the Chebyshev potentials.

Let us make two remarks. First, as a corollary of the above statement, the AskeyWilson potentials with parameters (8.14) can be represented as the determinant of a specific $M \times M$ matrix, $M=\sum n_{i}-2$, composed from solutions of the free DSE [53, 54]. Second, since all integers $n_{i}$ enter as free parameters, one can consider their continuation to arbitrary real (or complex) values, and this results in the completely general set of the Askey-Wilson potentials. Loosely speaking, this means that (8.2), (8.3), and thus all the associated polynomials, are built from the Chebyshev potentials/polynomials. This statement may be taken as a physical explanation of the existence of many nice properties of the system (8.1)-(8.5).

Comment. Evidently, all the potentials with parameters $a_{i} q^{n_{i}}$ are Darboux equivalent, and the choice (8.14) is distinguished by its simplicity. It is this Darboux equivalence that was used by Askey and Wilson [7] for calculation of the weight function for their polynomials. Namely, they used the fact that the homogeneous limit $n_{i} \rightarrow \infty$ in $a_{i} q^{n_{i}}$ 
sets all parameters equal to zero, which corresponds to the $q$-Hermite polynomials with known weight function. Moreover, the choice (8.11) was used for fixing the latter weight function in explicit form. In fact, even the specific character of the choice of parameters (8.14) was remarked on in the Addendum to the seminal memoir [7]. Therefore, Proposition 6 is just a reinterpretation of the results of [7]. One of the goals of the present paper was to uncover the discrete Darboux transformation behind these peculiarities and to show that the latter transformation is very useful for building more and more complicated discrete potentials from simple ones. We stress that the system of Askey-Wilson potentials is a very special class of potentials that can be obtained in this way. Starting from the free DSE, one can easily construct systems with a finite discrete spectrum of an arbitrary form.

In general, the potentials $u_{n}$ and $b_{n}$ defined by the choice (8.14) contain singularities. However, one can find a wide subclass of parameters (8.14) for which the following requirements are satisfied:

(i) $u_{n}>0$ for $-\infty<n<\infty$, and $u_{n} \rightarrow 1, b_{n} \rightarrow 0$ exponentially fast for $n \rightarrow \pm \infty$;

(ii) $u_{n}$ and $b_{n}$ do not have singularities for any integer $n$.

For such potentials, one can formulate the scattering problem on the whole infinite lattice $-\infty<n<\infty$. The specific property of potentials which are Darboux equivalent to the free ones is that they are reflectionless, i.e., a solution of the corresponding Schrödinger equation (in the self-adjoint form) with asymptotics $\chi_{n} \propto e^{i \theta n}$ at $n \rightarrow-\infty$ has analogous asymptotics, $\chi_{n} \propto e^{i \theta n}$, when $n \rightarrow \infty[1,76]$.

Let us present some explicit examples obtained after the repeated double Darboux transformations associated with the Volterra lattice.

(I) In [72], the reflectionless potentials

$$
u_{n}(j)=\frac{c_{n} c_{n+2 j+1}}{c_{n+j} c_{n+j+1}}, \quad b_{n}(j)=0,
$$

where $c_{n} \equiv \cosh \omega n, \omega>0$, were obtained by successive dressing of the free DSE with the help of auxiliary wave functions $\phi_{n}(j)=c_{n+1} c_{n+2} \cdots c_{n+j}$. Indeed, it is easy to see that $\phi_{n}(j)$ is a non-normalizable solution of DSE with potentials $u_{n}(j-1)$, $b_{n}(j-1)=0$ with the eigenvalue $\mu(j)=2 \cosh \omega j$. Potentials (8.15) appear from (8.2) after setting $a_{1}=-q^{-j / 2}, a_{2}=q^{1+j / 2}, a_{3}=q^{1 / 2-j / 2}, a_{4}=-q^{1 / 2+j / 2}$, and shifting $n \rightarrow n+j+1 / 2$. In $l^{2}(\mathbf{Z})$, they have a continuous spectrum in the interval $-2 \leq \lambda \leq 2$ and the discrete spectrum $\lambda_{k}= \pm 2 \cosh \omega k, k=1, \ldots, j$. In the infinite discrete spectrum limit $j \rightarrow \infty$, taken after the shift $n \rightarrow n-j$, they correspond to the system of $q^{-1}$-Hermite polynomials $[4,45,57,74]$.

(II) Another class of potentials,

$$
u_{n}(j)=\frac{s_{n} s_{n+2 j+1}}{s_{n+j} s_{n+j+1}}, \quad b_{n}(j)=0,
$$

where $s_{n} \equiv \sinh \omega n$, is obtained as a result of dressing free DSE by the functions $\phi_{n}(j)=s_{n+1} s_{n+2} \cdots s_{n+j}$. It corresponds to the parameters $a_{1}=-a_{2}=q^{(j+1) / 2}$, $a_{3}=-a_{4}=q^{1+j / 2}$. The potentials (8.16) cannot be said to be reflectionless due to the presence of singularities. In the limit $j \rightarrow \infty$, the singularities disappear and we arrive at the recurrence relation for the ordinary $q$-Hermite polynomials. Note that potentials (8.16) correspond to the particular class of $q$-ultraspherical polynomials [7], and the potentials (8.15) correspond to the associated $q$-ultraspherical polynomials.

(III) Perhaps the most interesting class of discrete potentials is obtained after Darboux transformations, defined by the trigonometric solutions of free DSE, $\phi_{n} \propto \sin \varphi n$, 
$\cos \varphi n$, when $\varphi$ is a fraction of $\pi$. The simplest single-Darboux-transformation example of this type has been considered in section 3 . The $j$-steps of similar DDT lead to the potentials:

$$
u_{n}(j)=\frac{\sin \pi n / N \sin \pi(n+2 j+1) / N}{\sin \pi(n+j) / N \sin \pi(n+j+1) / N}, \quad b_{n}(j)=0 .
$$

These are the trigonometric analogs of the potentials (8.16) with $\varphi=\pi / N$. As Askey informed us, such potentials also appeared in some unpublished notes by B. F. Logan. It is easily seen that

$$
\begin{gathered}
u_{0}(j)=u_{N-2 j-1}(j)=0, \\
u_{n}(j)>0, \quad 0<n<N-2 j-1,
\end{gathered}
$$

which shows that we are dealing with the finite-dimensional set of orthogonal polynomials. In fact, this system is very simple, and it is obtained from the finitedimensional Chebyshev polynomials of the second kind with the spectrum $2 \cos \pi k / N$, $k=1, \ldots, N-1$ by $j$-fold undressing of the first $j$ levels from below and $j$ levels from above. After that, the spectrum is $\lambda_{k}=2 \cos \pi k / N, k=j+1, \ldots, N-j-1$. It is obvious that the highest power of these polynomials is $N-2 j-1$.

Shifting $n \rightarrow n+N / 2$ in (8.17) and then replacing $N$ by $2 N$, we arrive at the potentials

$$
u_{n}=\frac{\cos \pi n / 2 N \cos \pi(n+2 j+1) / 2 N}{\cos \pi(n+j) / 2 N \cos \pi(n+j+1) / 2 N}, \quad b_{n}=0,
$$

which are root-of-unity partners of the potentials (8.15) for $\varphi=\pi / 2 N$. For arbitrary $N>j+1$, we see that $u_{-N}=u_{N-2 j-1}=0$ and $u_{n}>0$ are between these zeros.

Using the correspondence between solutions of the Toda and Volterra lattices (see, e.g., $[35,72])$, it is possible to generate non-trivial root-of-unity systems for which $u_{n} \geq 0$ and $b_{n} \neq 0$. More precisely, for any two-diagonal Hamiltonian

$$
H_{V}|n\rangle=|n+1\rangle+u_{n}^{V}|n-1\rangle,
$$

one can associate the three-diagonal one, $H_{T}=H_{V}^{2}-2$

$$
H_{T}|n\rangle=|n+2\rangle+u_{n}^{V} u_{n-1}^{V}|n-2\rangle+\left(u_{n}^{V}+u_{n+1}^{V}-2\right)|n\rangle .
$$

We subtract 2 from $H_{V}^{2}$ in order for both operators $H_{V}$ and $H_{T}$ to have the continuous spectrum band $-2 \leq \lambda \leq 2$ (if it exists). Looking now at the lattice formed by the sites with odd (or even) index label and renormalizing the lattice step to be 1 , one gets a Hamiltonian (1.8) with potentials $u_{n}^{T}=u_{2 n}^{V} u_{2 n+1}^{V}, b_{n}^{T}=u_{2 n+1}^{V}+u_{2 n+2}^{V}-2$. For example, the Toda-analog of the potentials (8.17) after replacing $N$ by $2 N$ will be

$$
\begin{aligned}
& u_{n}=\frac{s_{n} s_{n+1 / 2} s_{n+j+1 / 2} s_{n+j+1}}{s_{n+j / 2} s_{n+j / 2+1} s_{n+j / 2+1 / 2}^{2}}, \\
& b_{n}=-\frac{2 \cos \pi / 2 N s_{j / 2} s_{j / 2+1 / 2}}{s_{n+j / 2+1 / 2} s_{n+j / 2+3 / 2}},
\end{aligned}
$$

where $s_{n}=\sin \pi n / N$. The potential $u_{n}$ is positive between the zeros at $n=0$ and $n=N-j-1$. The spectrum is $\lambda_{m}=2 \cos \pi m / N, m=j+1, \ldots, N-1$, i.e., the dimension of the system is $N-j-1$. 
The two-parametric set of similar potentials is obtained by removing additional $k$ levels from above

$$
\begin{gathered}
u_{n}(j, k)=\frac{s_{n} s_{n+j+k+1} s_{n+j+1 / 2} s_{n+k+1 / 2}}{s_{n+(j+k+1) / 2}^{2} s_{n+(j+k) / 2} s_{n+1+(j+k) / 2}}, \\
b_{n}(j, k)=\frac{2 \cos \pi / 2 N s_{(k-j) / 2} s_{(k+j+1) / 2}}{s_{n+(j+k+1) / 2} s_{n+(j+k+3) / 2}} .
\end{gathered}
$$

The corresponding polynomials are natural discrete analogs of the Jacobi polynomials $P_{n}^{(j+1 / 2, k+1 / 2)}(x)$, which appear in the limit $N \rightarrow \infty$. Note that the case $j=k$ corresponds to (8.17), whereas $k=0$ corresponds to $(8.20),(8.21)$.

Proposition 7. The weight function for the two-parameter set of orthogonal polynomials generated by (8.22), (8.23) is

$$
w\left(x_{m} ; j, k\right)=\sin ^{2} \frac{\pi m}{N} \prod_{l=1}^{j}\left(\cos \frac{\pi l}{N}-x_{m}\right) \prod_{s=1}^{k}\left(\cos \frac{\pi s}{N}+x_{m}\right)
$$

where $x_{m}=\cos \pi m / N, m=j+1, \ldots, N-k-1$. I.e., it consists of a reduced number of discrete jumps of the finite-dimensional Chebyshev polynomials.

This statement follows from the fact that successive removal of levels is equivalent to changes to kernel polynomials, whose weight function differs from the original one only by a polynomial factor determined by the zeros of finite-dimensional Chebyshev polynomials.

The examples presented above do not exhaust all possible positive root-of-unity potentials, e.g., one can generate a three-parameter set of analogous potentials for odd $N$ by symmetric removal of $2 r$ levels from the middle of the spectrum with the help of the double DDT for the Volterra chain, etc. Properties of the corresponding $q$-orthogonal polynomials do not seem to be analyzed in the literature. The authors have some results on this subject, but it is appropriate to present them separately. The richness of the situation is demonstrated by the following.

Proposition 8. Recurrence coefficients of the q-ultraspherical polynomials

$$
u_{n}=\frac{\sin \varphi n \sin \varphi(n+2 \alpha+1)}{\sin \varphi(n+\alpha) \sin \varphi(n+\alpha+1)}, \quad \varphi=\pi p / N
$$

where $\alpha$ is a real parameter, are positive for integer values of $n$ between two successive zeros at $n=0$ and $n=s>0$ when

(1) $p=1$ and $-3 / 2<\alpha<-1,-1<\alpha<0,0<\alpha<1 / 2, s=N$;

(2) $p=1$ and $\alpha=\frac{1}{2}, \frac{3}{2}, \ldots,\left[\frac{N-1}{2}\right]-\frac{1}{2}, s=N-2 \alpha-1$;

(3) $p=1, N-1$ and $\alpha=1,2, \ldots, N / 2-1, s=N-2 \alpha-1, N$ even;

(4) $p=1,(N-1) / 2,(N+1) / 2, N-1$ and $\alpha=1,2, \ldots,(N-3) / 2, s=N-2 \alpha-1$, $N$ odd.

Another problem which is worth pursuing is the classification of all reflectionless potentials of the Askey-Wilson type and analysis of the limits of an infinite number of DDT [72].

Finally, let us discuss briefly the dynamical symmetries. As found in [72], the strange series representation of the quantum algebra $s u_{q}(1,1)$ in Cartesian form corresponds to the potentials (8.15). The compact analog of the same quantum algebra 
describes symmetries of (8.17). Indeed, one can verify that the abstract Hermitian operators $K_{0}, K_{1}$ given by

$$
\begin{gathered}
K_{0}|n\rangle=\frac{\cos \pi(n+j+1) / N}{\sin \pi / N}|n\rangle, \\
K_{1}|n\rangle=\frac{\sqrt{u_{n+1}(j)}}{2 \sin \pi / N}|n+1\rangle+\frac{\sqrt{u_{n}(j)}}{2 \sin \pi / N}|n-1\rangle,
\end{gathered}
$$

where $u_{n}(j)$ is fixed in (8.17), satisfy the relations defining the Cartesian form of the $s u_{q}(2)$ algebra:

$$
\begin{aligned}
& p^{-1} K_{0} K_{1}-p K_{1} K_{0}=i K_{2}, \\
& p^{-1} K_{2} K_{0}-p K_{0} K_{2}=i K_{1}, \\
& p^{-1} K_{1} K_{2}-p K_{2} K_{1}=i K_{0}
\end{aligned}
$$

where $K_{2}$ is defined by the first equality, and $p \equiv e^{-i \pi / 2 N}=q^{1 / 4}$ (for the nonlinear map of generators of the standard raising and lowering operator form of $s u_{q}(2)$ onto (8.27), see [88]). Note that for the choice $j=0$, the states $|n\rangle$ correspond to finitesize Chebyshev polynomials, i.e., the latter provide the simplest examples of $q$-special functions for $q$ equal to a root of 1 . Similarly one can consider the complementary series representation of the given algebra containing a free continuous parameter and find other types of positive potentials (8.25).

Acknowledgments. The authors are deeply indebted to R. Askey for illuminating conversations and helpful remarks, to B. A. Kupershmidt for stimulating communications, to T. S. Chihara, F. Gesztesy, M. E. H. Ismail, F. Marcellán, and D. R. Masson for sending their papers, and to M. Rahman, S. K. Suslov, and L. Vinet for helpful discussions. We would like to thank the Centre de Recherches Mathématiques of the Université de Montréal for its hospitality, and the support of the visit of AZh to the Centre in January 1994, during which time this paper was basically completed. The work of VS was supported by NSERC (Canada) and by Fonds FCAR (Québec). The work of AZh was supported in part by Grant No. U9E000 from the International Science Foundation.

\section{References}

1. M. J. Ablowitz and H. Segur, Solitons and the Inverse Scattering Transform, SIAM, Philadelphia, 1981 .

2. M. Adler and J. Moser, On a class of polynomials connected with the Korteweg-de Vries equation, Comm. Math. Phys. 61 (1978), 1-30.

3. W. Al-Salam, W. R. Allaway, and R. Askey, Sieved ultraspherical polynomials, Trans. Amer. Math. Soc. 284 (1984), 39-55.

4. R. Askey, Continuous $q$-Hermite polynomials when $q>1$, In: $q$-Series and Partitions (D. Stanton, ed.), IMA Volumes in Mathematics and Its Applications, Springer-Verlag, New York, (1989), 151-158.

5. R. Askey and M. Ismail, Recurrence relations, continued fractions and orthogonal polynomials, Mem. Amer. Math. Soc. 49 (1984), 1-107.

6. R. Askey and J. Wilson, A set of orthogonal polynomials that generalize the Racah coefficients or $6 j$-symbols, SIAM J. Math. Anal. 10 (1979), 1008-1016.

7. Mem. Amer. Math. Soc. 54 (1985), 1-55.

8. R. Baxter, Exactly Sovable Models in Statistical Mechanics, Academic Press, 1982.

9. M. Bruschi, O. Ragnisco, P. M. Santini, and G.-Z. Tu, Integrable symplectic maps, Physica D49 (1991), 273-294. 
10. J. L. Burchnall and T. W. Chaundy, Commutative ordinary differential operators, Proc. Lond. Math. Soc. 21 (1923), 420-440.

11. J. Bustoz, M. E. H. Ismail, and J. Wimp, On sieved orthogonal polynomials VI: differential equations, Diff. Int. Eqs. 13 (1990), 757-766.

12. K. M. Case, Orthogonal polynomials from the viewpoint of scattering theory, J. Math. Phys. 15 (1974), 2166-2174.

13. J. A. Charris and M. E. H. Ismail, Sieved orthogonal polynomials. VII: generalized polynomial mappings, Trans. Amer. Math. Soc. 340 (1993), 71-93.

14. L. M. Chihara, Askey-Wilson polynomials, kernel polynomials, and association schemes, Graphs and Combin. 9 (1993), 213-223.

15. L. M. Chihara and T. S. Chihara, A class of nonsymmetric orthogonal polynomials, J. Math. Anal. Appl. 126 (1987), 275-291.

16. T. S. Chihara, On co-recursive orthogonal polynomials, Proc. Amer. Math. Soc. 8 (1957), 899905.

17. An Introduction to Orthogonal Polynomials, Gordon and Breach, 1978.

18. __ Orthogonal polynomials and measures with end point masses, Rocky Mountain J. Math. 15 (1985), 705-719.

19. D. V. Chudnovsky, Topological and algebraic structure of linear problems associated with completely integrable systems, In: Lecture Notes in Physics 180, Springer-Verlag, Berlin, 1983, 65-90.

20. D. V. Chudnovsky and G. V. Chudnovsky, Laws of composition of Bäcklund transformations and the universal form of completely integrable systems in dimensions two and three, Proc. Nat. Acad. Sci. U.S.A. 80 (1983), 1774-1777.

21. M. M. Crum, Associated Sturm-Liouville systems, Quart. J. Math. 6 (1955), 121-127.

22. G. Darboux, Lecons sur la Théorie Génerale des Surfaces et les Applications Géométrique du Calcul Infinitésimal, Paris, Gauthier Villars et Fils (1889), 2, Entry 408, 196.

23. P. Deift, Applications of a commutation formula, Duke Math. J. 45 (1978), 267-310.

24. J. J Duistermaat and F. A. Grünbaum, Differential equations in the spectral parameter, Comm. Math. Phys. 103 (1986), 177-240.

25. A. Erdélyi, W. Magnus, F. Oberhettinger, and F. G. Tricomi, Higher Transcendental Functions, Bateman Manuscript Project Vol. 2, McGraw-Hill, 1953.

26. R. Floreanini, J. LeTourneux, and L. Vinet, Quantum mechanics and polynomials of a discrete variable, Ann. Phys. 226 (1993), 331-349.

27. D. Galant, An implementation of Christoffel's theorem in the theory of orthogonal polynomials, Math. Comp. 25 (1971), 111-113.

28. G. Gasper and M. Rahman, Basic Hypergeometric Series, Cambridge University Press, Cambridge, 1990.

29. W. Gautschi and $\mathrm{S}$. Li, A set of orthogonal polynomials induced by a given orthogonal polynomial, Aequ. Math. 46 (1993), 174-198.

30. J. S. Geronimo, Scattering theory, orthogonal polynomials, and q-series, SIAM J. Math. Anal. 25 (1994), 392-419.

31. J. S. Geronimo and K. M. Case, Scattering theory and polynomials orthogonal on the real line, Trans. Amer. Math. Soc. 258 (1978), 467-494.

32. J. S. Geronimo and W. Van Assche, Orthogonal polynomials on several intervals via a polynomial mapping, Trans. Amer. Math. Soc. 308 (1988), 559-581.

33. J. Geronimus, On polynomials orthogonal with regard to a given sequence of numbers, Zapiski Nauchno-Issled. Inst. Mat. i Mehan. Har'kov. Univ. i Har'kov. Mat. Obsch. Ser. 417 (1940), 3-18 (in Russian).

34. On polynomials orthogonal with respect to a given sequence of numbers and a theorem by W. Hahn, Izv. Acad. Sci. USSR 4 (1940), 215-228.

35. F. Gesztesy, H. Holden, B. Simon, and Z. Zhao, On the Toda and Kac-van Moerbeke systems, Trans. Amer. Math. Soc. 339 (1993), 849-868.

36. F. Gesztesy, W. Karwowski, and Z. Zhao, Limits of soliton solutions, Duke Math. J. 68 (1992), 101-150.

37. F. Gesztesy and R. Svirsky, $(m) K d V$-solitons on the background of quasi-periodic finite-gap solutions, preprint (1991).

38. J. Gibbons and B. A. Kupershmidt, Time discretizations of lattice integrable systems, Phys. Lett. A165 (1992), 105-110.

39. E. Godoy and F. Marcellán, An analog of the Christoffel formula for polynomial modification of a measure on the unit circle, Boll. U.M.I. 5A (1991), 1-12. 
40. Ya. I. Granovskii and A. S. Zhedanov, Orthogonal polynomials on Lie algebras, Izvestiya Vuzov (Fizika) 5 (1986), 60-66 (in Russian).

41. D. P. Gupta and D. R. Masson, Exceptional $q$-Askey-Wilson polynomials and continued fractions, Proc. Amer. Math. Soc. 112 (1991), 717-727.

42. R. Hirota, Nonlinear partial difference equations II. Discrete time Toda equation, J. Phys. Soc. Japan 43 (1977), 2074-2078.

43. R. Hirota, S. Tsujimoto, and T. Imai, Difference scheme of soliton equations, In: Proceedings of "Future Directions of Nonlinear Dynamics in Physical and Biological Systems" (P. L. Christiansen, J. C. Eilbeck, and R. D. Parmentier, eds.), Plenum Press, 1993, 7-15.

44. L. Infeld and T. E. Hull, The factorization method, Rev. Mod. Phys 23 (1951), 21-68.

45. M. E. H. Ismail and D. R. Masson, q-Hermite polynomials, biorthogonal rational functions, and q-beta integrals, Trans. Amer. Math. Soc. 346 (1994), 63-116.

46. M. E. H. Ismail and M. Rahman, The associated Askey-Wilson polynomials, Trans. Amer. Math. Soc. 328 (1991), 201-237.

47. M. E. H. Ismail and R. W. Ruedemann, Relation between polynomials orthogonal on the unit circle with respect to different weights, J. Approx. Theory 71 (1992), 39-60.

48. M. G. Krein, On the continuous analog of the Christoffel formula in the theory of orthogonal polynomials, Dokl. Acad. Nauk USSR 113 (1957), 970-973 (in Russian).

49. I. M. Krichever, Algebraic curves and nonlinear difference equations, Russian Math. Surveys 33 (1978), 255-256.

50. D. Levi, Nonlinear differential-difference equations as Bäcklund transformations, J. Phys. A14 (1981), 1083-1098.

51. D. Levi and P. Winternitz, Continuous symmetries of discrete equations, Phys. Lett. A152 (1991), 335-338.

52. D. R. Masson and J. Repka, Spectral theory of Jacobi matrices in $l^{2}(Z)$ and the su( 1,1$)$ Lie algebra, SIAM J. Math. Anal. 22 (1991), 1131-1146.

53. V. B. Matveev, Darboux transformation and explicit solutions of differential-difference and difference-difference evolution equations I, Lett. Math. Phys. 3 (1979), 217-222.

54. V. B. Matveev and M. A. Salle, Differential-difference evolution equations II (Darboux transformation for the Toda lattice), Lett. Math. Phys. 3 (1979), 425-429.

55. W. Miller, Jr., Lie theory and difference equations I, J. Math. Anal. Appl. 28 (1969), 383-399.

56. F. W. Nijhoff, V. G. Papageorgiou, H. W. Capel, and G. R. W. Quispel, The lattice Gelfand-Dikii hierarchy, Inverse Problems 8 (1992), 597-621.

57. A. F. Nikiforov and S. K. Suslov, Classical orthogonal polynomials of a discrete variable on nonuniform lattices, Lett. Math. Phys. 11 (1986), 27-34.

58. A. F. Nikiforov, S. K. Suslov, and V. B. Uvarov, Classical Orthogonal Polynomials of a Discrete Variable, Springer, 1991.

59. V. Yu. Novokshenov, Reflectionless potentials and soliton series of the KdV equation, Teor. Mat. Fiz. 92 (1992), 286-301.

60. V. Papageorgiou, Integrable nonlinear partial difference equations and numerical analysis algorithms, talk presented at the Workshop on Symmetries and Integrability of Difference Equations (Estérel, Canada, May 22-29, 1994).

61. G. R. W. Quispel, J. A. G. Roberts, and C. J. Thompson, Integrable mappings and soliton equations, Phys. Lett. A126 (1988), 419-421.

62. E. Schrödinger, A method of determining quantum mechanical eigenvalues and eigenfunctions, Proc. Royal Irish Academy A46 (1940), 9-16.

63. __ Further studies on solving eigenvalue problems by factorization, Proc. Royal Irish Academy A46 (1940), 183-206.

64. A. Shabat, The infinite dimensional dressing dynamical system, Inverse Problems 8 (1992), 303308.

65. A. B. Shabat and R. I. Yamilov, Theory of nonlinear chains, Leningrad Math. J. 2 (1991), $377-400$.

66. S. Skorik and V. Spiridonov, Self-similar potentials and the q-oscillator algebra at roots of unity, Lett. Math. Phys. 28 (1993), 59-74.

67. K. Sogo, Toda molecule equation and quotient-difference method, J. Phys. Soc. Japan 62 (1993), 1081-1084.

68. V. Spiridonov, Universal superpositions of coherent states and self-similar potentials, Phys. Rev. A, to appear. 
69. __ Exactly solvable potentials and quantum algebras, Phys. Rev. Lett. 69(1992), 398-401; Proc. of the XIXth ICGTMP vol. I, (M. A. del Olmo, M. Santander, and J. M. Guilarte, eds.), 198-201, 1992.

70. V. Spiridonov, L. Vinet, and A. Zhedanov, Difference Schrödinger operators with linear and exponential discrete spectra, Lett. Math. Phys. 29 (1993), 63-73.

71. _ Periodic reductions of the factorization chain and the Hahn polynomials, J. Phys. A: Math. Gen. 27 (1994), L669-L675.

72. V. Spiridonov and A. Zhedanov, Discrete reflectionless potentials, quantum algebras, and $q-$ orthogonal polynomials, Ann. Phys. 237 (1995), 126-146.

73. Yu. B. Suris, Discrete time generalized Toda lattices: complete integrability and relation with relativistic Toda lattices, Phys. Lett. A145 (1990), 113-119.

74. S. K. Suslov, Classical orthogonal polynomials of a discrete variable continuous orthogonality relation, Lett. Math. Phys. 14 (1987), 77-88.

75. G. Szegö, Orthogonal Polynomials, AMS, 1959.

76. M. Toda, Theory of Nonlinear Lattices, Springer, 1981.

77. W. Van Assche and A. P. Magnus, Sieved orthogonal polynomials and discrete measures with jumps dense in an interval, Proc. Amer. Math. Soc. 106 (1989), 163-173.

78. J. F. Van Diejen, Families of Commuting Difference Operators, PhD. Thesis, University of Amsterdam, 1994.

79. S. Venakides, P. Deift, and R. Oba, The Toda shock problem, Comm. Pure Appl. Math. 44 (1991), 1171-1242.

80. A. P. Veselov, Integrable maps, Russian Math. Surveys 46 (1991), 3-45.

81. A. P. Veselov and A. B. Shabat, Dressing chain and spectral theory of Schrödinger operator, Funkt. Anal. i evo Pril. 27 n2 (1993), 1-21.

82. V. B. Uvarov, The connection between systems of polynomials orthogonal with respect to different distribution functions, U.S.S.R. Comp. Math. \& Math. Phys. 9 (1969), 25-36.

83. J. H. Wilkinson, The Algebraic Eigenvalue Problem, Clarendon Press, Oxford, 1965.

84. B. N. Zakhariev, Discrete and continuous quantum mechanics. Exactly solvable models (Lessons of quantum intuition II), Sov. J. Part. Nucl. 23 (1992), 603-640.

85. V. E. Zakharov and A. B. Shabat, A scheme for integrating the nonlinear equations of mathematical physics by the method of the inverse scattering problem I, Funct. Anal. Appl. 8 (1974), 226-235.

86. - Integration of nonlinear equations of mathematical physics by the method of inverse scattering II, Funct. Anal. Appl. 13 (1979), 166-174.

87. A. S. Zhedanov, The Toda chain: solutions with dynamical symmetry and classical orthogonal polynomials, Theor. Math. Phys. 82 (1990), 6-11.

88. ㄴ_ Quantum $\mathrm{su}_{q}(2)$ algebra: “Cartesian” version and overlaps, Mod. Phys. Lett. A7 (1992), 2589-2593.

Centre de Recherches Mathématiques, Université de Montréal, C.P. 6128, Succ. Centreville, Montréal, QuÉBEc, H3C 3J7, CANAdA (On leave from the Institute for Nuclear Research, Russian Academy of Sciences, Moscow, Russia)

E-mail: spiridonov@lps.umontreal.ca

Physics Department, Donetsk University, Donetsk, 340055, Ukraine 